\title{
Nonlinear frequency response analysis of structural vibrations
}

\author{
O. Weeger · U. Wever · B. Simeon
}

Received: date / Accepted: date

\begin{abstract}
In this paper we present a method for nonlinear frequency response analysis of mechanical vibrations of 3dimensional solid structures. For computing nonlinear frequency response to periodic excitations, we employ the wellestablished harmonic balance method. A fundamental aspect for allowing a large-scale application of the method is model order reduction of the discretized equation of motion. Therefore we propose the utilization of a modal projection method enhanced with modal derivatives, providing second-order information. For an efficient spatial discretization of continuum mechanics nonlinear partial differential equations, including large deformations and hyperelastic material laws, we use the isogeometric finite element method, which has already been shown to possess advantages over classical finite element discretizations in terms of higher accuracy of numerical approximations in the fields of linear vibration and static large deformation analysis. With several computational examples, we demonstrate the applicability and accuracy of the modal derivative reduction method for nonlinear static computations and vibration analysis. Thus, the presented method opens a promising perspective on application of nonlinear frequency analysis to large-scale industrial problems.
\end{abstract}

Keywords Nonlinear vibration $\cdot$ model reduction $\cdot$ modal derivatives $\cdot$ harmonic balance $\cdot$ isogeometric analysis

Oliver Weeger · Utz Wever

Siemens AG, Corporate Technology

Otto-Hahn-Ring 6, 81739 Munich, Germany

E-mail: oliver.weeger.ext@siemens.com,

E-mail: utz.wever@siemens.com

Oliver Weeger · Bernd Simeon

TU Kaiserslautern, Faculty of Mathematics,

P.O. Box 3049, 67653 Kaiserslautern, Germany

E-mail: weeger@rhrk.uni-kl.de

E-mail: simeon@mathematik.uni-kl.de

\section{Introduction}

Vibration analysis and nonlinear structural analysis both play an important role in the industrial mechanical engineering process, but so far there are no efficient methods available for a nonlinear structural frequency response analysis on a large scale.

For nonlinear frequency response analysis we use the harmonic balance method (HBM) [1-3], which transforms and solves the underlying equation of motion in the frequency domain. In previous work we have already investigated nonlinear structural vibrations with isogeometric finite elements and harmonic balance, and have demonstrated its applicability using the nonlinear Euler-Bernoulli beam structural model [4]. Now we extended this method to $3-$ dimensional nonlinear structural mechanics with large deformations and hyperelastic material laws [5,6].

Though harmonic balance is a well-established method for nonlinear frequency analysis, for example in the context of integrated circuit simulations [7,8], it is so far hardly used in mechanics, only for lower dimensional structural models such as beams and plates [9,-13]. Commerical finite element analysis (FEA) software such as ANSYS, Nastran or ABAQUS do not provide any methods dedicated to nonlinear frequency response. This is mainly due to the truncated Fourier expansion HBM uses for frequency domain approximation of each degree of freedom (DOF) of the spatial discretization, which produces a blow-up of total DOFs: the sparse linear system to be solved in the end is not only $m$ times bigger, but also with $m$-times as many non-zero entries per row as the spatial discretization, where $m$ is the number of Fourier coefficients.

Therefore we need model order reduction (MOR) of the spatial discretization to reduce the size of the linear system significantly and make an efficient numerical solution of the system arising from HBM even possible. 
While modal reduction, where the equation of motion is projected onto a subspace spanned by a selection of eigenmodes, is a well-established technique in linear FEA and vibration analysis [14], more advanced methods are needed in the nonlinear context [5 15]. For example in [16] a singleDOF reduction on nonlinear modes was introduced.

We propose to use a modal reduction with modal derivatives [17, 18], which are a second-order enhancement of linear eigenmodes. The method has been successfully applied in nonlinear dynamic analysis by time-integration before [19. [22], and we show that it is especially suitable in our nonlinear vibration framework with harmonic balance. In contrast to most other common reduction methods so far used in nonlinear time-integration, it does not require a current state of deformation of the system and continuous basis updates, thus the projection basis can be fully pre-computed based on the linear system. Furthermore there are also similar well-established techniques of second-order enhancements in other fields of computational engineering such as uncertainty quantification [23].

For the spatial discretization we rely on the isogeometric finite element method, but note that the proposed nonlinear frequency analysis method with modal derivative reduction could be applied using any spatial discretization method. Isogeometric analysis (IGA) was introduced by Hughes et al. [24] in 2005 and aims at closing the gap between computeraided design (CAD), numerical simulation and manufacturing (CAM) by using the same geometry representation throughout the whole engineering process. As spline functions, such as B-Splines and non-uniform rational B-Splines (NURBS) [25], are typically used for geometry design in CAD software, in IGA these functions are also employed for discretization of geometry and numerical solution in an isoparameteric fashion. This concept has already been successfully applied to several numerical discretization methods, such as boundary elements, collocation, finite volumes, and, of course, isogeometric finite elements [24, 26-28]. A detailed introduction into IGA and collection of numerical analysis, properties and applications of the method can be found in the monograph [29].

It has been shown that isogeometric finite elements have substantial advantages over classical Lagrangian finite elements in the context of linear vibration analysis, i.e. solution of eigenvalue problems, where so-called optic and acoustic branches are avoided, which leads to a much higher accuracy especially in higher eigenfrequencies [30]. In general, IGA provides higher accuracy per DOF for numerical solution of linear elliptic, parabolic and hyperbolic partial differential equations (PDEs) than standard finite element methods due to higher continuity of splines, whereas rates of convergence are the same [29,31]. The method has also been applied in nonlinear continuum mechanics, where the advantages of the approach could be verified [32-34].
The further structure of this paper after after this introductory Section 1 is as follows: We continue with a summary of continuum mechanics equations of large deformation hyperelasticity and their isogeometric finite element discretization in Section 2 Then we give a brief review of modal analysis and direct frequency response as means of linear vibration analysis in Section 3 , before we introduce the harmonic balance method in application to nonlinear structural frequency response. Section 4 is dedicated to model order reduction methods, with a review of commonly used methods followed by a detailled introduction into modal reduction with the concept of modal derivatives. With the computational examples presented in Section 5, we prove the functioning of our framework for nonlinear structural vibration analysis and show that it suitable for large-scale applications. Then we conclude with a short summary of the work presented and give an outlook on future research directions in Section 6 .

\section{Isogeometric finite element discretization of large deformation hyperelasticity}

In this work we address the numerical simulation of dynamic behaviour of mechanical structures described by geometrical and material nonlinearities. Therefore in this section we give a summary of the theory of continuum mechanics with large deformation kinematics and constitutive laws of hyperelasticity. Then we derive the spatial discretization of governing equations using isogeometric finite elements.

\subsection{Kinematics and constitutive laws}

First we want to give a brief review of the Total Lagrangian formulation of kinematics and constitutive relations of solids subject to large deformations and hypererlastic material behaviour, based on the monographs [5, 6].

In the Total Lagrangian point of view, motion and deformation of a body over time are described with respect to its initial configuration given by the domain $\Omega \in \mathbb{R}^{3}$. At every time $t$ in the interval of interest $[0, T]$, the current position $\mathbf{x} \in \Omega_{t} \subset \mathbb{R}^{3}$ of each point $\mathbf{X} \in \Omega$ can be expressed in terms of its initial position and a displacement field $\mathbf{u} \in \mathbb{R}^{3}$ (see also Figure 1):

$\mathbf{x}(\mathbf{X}, t)=\mathbf{X}+\mathbf{u}(\mathbf{X}, t)$

For the description of the deformation process we need the deformation gradient, i.e. the spatial gradient of current w.r.t. to initial position of each point:

$\mathbf{F}(\mathbf{X}, t)=\frac{d \mathbf{x}}{d \mathbf{X}}(\mathbf{X}, t)=\mathbf{I}+\frac{d \mathbf{u}}{d \mathbf{X}}(\mathbf{X}, t)=\mathbf{I}+\nabla \mathbf{u}(\mathbf{X}, t)$. 


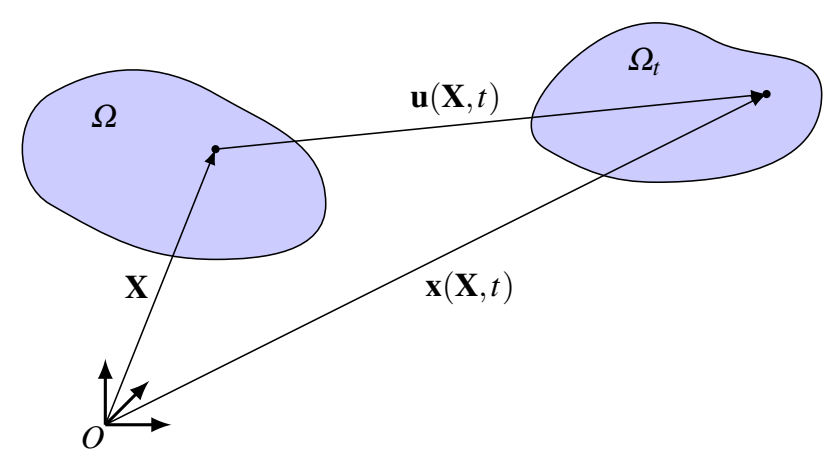

Fig. 1: Motion of the body with domain $\Omega$

The Jacobian determinant $J=\operatorname{det} \mathbf{F}$ is a measure of the volume change of the body. For incompressible materials, which are not subject of this work, it holds $J=1$.

Furthermore we need a strain measure defined in the initial configuration, the Green-Lagrange strain tensor

$\mathbf{E}(\mathbf{X}, t)=\frac{1}{2}(\mathbf{C}(\mathbf{X}, t)-\mathbf{I})$.

It is defined using the right Cauchy-Green tensor

$\mathbf{C}(\mathbf{X}, t)=\mathbf{F}^{T} \mathbf{F}=\mathbf{I}+\nabla \mathbf{u}^{T}+\nabla \mathbf{u}+\nabla \mathbf{u}^{T} \nabla \mathbf{u}$,

which is a quadratic expression in terms of displacements resp. the deformation gradient. In linear elasticity theory the higher order term is ommitted and the linear strain measure is used:

$\mathbf{e}(\mathbf{X}, t)=\frac{1}{2}\left(\nabla \mathbf{u}^{T}+\nabla \mathbf{u}\right)$.

Velocity and acceleration of a point in the reference configuration are given as:

$\mathbf{v}(\mathbf{X}, t)=\dot{\mathbf{x}}(\mathbf{X}, t)=\frac{d \mathbf{x}}{d t}(\mathbf{X}, t)=\dot{\mathbf{u}}(\mathbf{X}, t)$,

$\mathbf{a}(\mathbf{X}, t)=\ddot{\mathbf{x}}(\mathbf{X}, t)=\frac{d^{2} \mathbf{x}}{d t^{2}}(\mathbf{X}, t)=\ddot{\mathbf{u}}(\mathbf{X}, t)$.

As stress measure in the material configuration we use the second Piola-Kirchoff stress tensor $\mathbf{S}$, which is related to the true Cauchy stress $\sigma$ in the current configuration by the following equation:

$\mathbf{S}=J \mathbf{F}^{-1} \sigma \mathbf{F}^{-T}$.

In hyperelasticity the constitutive relation of strain and stress is defined by a strain energy function $\psi$ :

$\mathbf{S}=\frac{d \psi}{d \mathbf{E}}=2 \frac{d \psi}{d \mathbf{C}}$.

In this work we refer to two particular choices of strain energy functions. For the linear St. Venant-Kirchhoff material law, which is also used in linear elasticty, it is

$$
\begin{aligned}
\psi(\mathbf{E}) & =\frac{\lambda}{2} \operatorname{tr}(\mathbf{E})^{2}+\mu \operatorname{tr}\left(\mathbf{E}^{2}\right), \\
\mathbf{S} & =\lambda \operatorname{tr}(\mathbf{E}) \mathbf{I}+2 \mu \mathbf{E},
\end{aligned}
$$

and for the nonlinear Neo-Hooke material it holds

$$
\begin{aligned}
\psi(\mathbf{C}) & =\frac{\lambda}{2}(\ln J)^{2}-\mu \ln J+\frac{\mu}{2}(\operatorname{tr}(\mathbf{C})-3), \\
\mathbf{S} & =\lambda \ln J \mathbf{C}^{-1}+\mu\left(\mathbf{I}-\mathbf{C}^{-1}\right) .
\end{aligned}
$$

For linearization within the later described solution process we are going to need the constitutive 4th order tensors

$\mathbf{C}^{S E}=\frac{d \mathbf{S}}{d \mathbf{E}}=2 \frac{d \mathbf{S}}{d \mathbf{C}}$

for both material laws. For the St. Venant-Kirchhoff material it is

$\mathbf{C}_{i j k l}^{S E}=\lambda \delta_{i j} \delta_{k l}+\mu\left(\delta_{i k} \delta_{j l}+\delta_{i l} \delta_{k j}\right)$,

and for the Neo-Hooke material

$\mathbf{C}_{i j k l}^{S E}=\lambda \mathbf{C}_{i j}^{-1} \mathbf{C}_{k l}^{-1}+(\mu-\lambda \ln J)\left(\mathbf{C}_{i k}^{-1} \mathbf{C}_{j l}^{-1}+\mathbf{C}_{i l}^{-1} \mathbf{C}_{k j}^{-1}\right)$.

\subsection{Strong and weak form of governing equations}

With the kinematic quantities introduced in the preceding Section 2.1, we can follow [5, 6] in formulating the local balance differential equations. In the strong form these must hold for all material points $\mathbf{X} \in \Omega$ and times $t \in[0, T]$.

The conservation of mass in the Lagrangian configuration reads as

$\rho J=\rho_{0}$,

where $\rho_{0}$ is the initial and $\rho$ the current mass density. Furthermore we need the conservation of linear momentum, involving volume forces $\rho_{0} \mathbf{b}$ :

$\operatorname{div} \mathbf{F ~ S}+\rho_{0} \mathbf{b}=\rho_{0} \ddot{\mathbf{u}}$.

Local balance of angular momentum yields the symmetry of the second Piola-Kirchhoff stress tensor

$\mathbf{S}=\mathbf{S}^{T}$

and the first law of thermodynamics, i.e. conservation of energy, reads:

$\rho_{0} \dot{u}=\mathbf{S} \cdot \dot{\mathbf{E}}-\operatorname{div} \mathbf{Q}+\rho_{0} R$,

where $u$ is the specific internal energy, $R$ the heat source and $\mathbf{Q}$ heat flux.

In addition to these equilibrium equations, we need boundary conditions for displacements and tractions:

$\mathbf{u}=\mathbf{u}_{d}$ on $\Gamma_{u}, \forall t \in[0, T]$,

$\mathbf{F} \mathbf{S} \mathbf{N}=\mathbf{t}$ on $\Gamma_{n}, \forall t \in[0, T]$,

where $\Gamma_{u}, \Gamma_{n} \subset \partial \Omega$ are the parts of the boundary of the domain $\Omega$ where prescribed displacements $\overline{\mathbf{u}}$ and $\overline{\mathbf{t}}$ tractions 
act, and $\mathbf{N}$ is the outer surface normal of a boundary point. For the time-dependent dynamic problem we also need the initial conditions of displacements and velocities

$\mathbf{u}(\mathbf{X}, 0)=\hat{\mathbf{u}}, \mathbf{v}(\mathbf{X}, 0)=\hat{\mathbf{v}} \quad \forall \mathbf{X} \in \Omega$.

In order to find an approximate solution of the exact displacements $\mathbf{u}$, we only demand that the equilibrium equations are fulfilled in a weak sense. Thus the residual remaining in (15) is multiplied with a test function $\delta \mathbf{u}$, the socalled virtual displacement fulfilling the boundary condition $\delta \mathbf{u}=\mathbf{0}$ on $\Gamma_{u}$, and then integrated over the domain $\Omega$. After a few manipulations this principle of virtual work yields the weak form of our problem:

$$
\begin{aligned}
\int_{\Omega} \rho_{0} \delta \mathbf{u}^{T} \ddot{\mathbf{u}} d \mathbf{X}+\int_{\Omega} \delta E \cdot \mathbf{S} d \mathbf{X} \\
=\int_{\Omega} \rho_{0} \delta \mathbf{u}^{T} \mathbf{b} d \mathbf{X}+\int_{\Gamma_{n}} \delta \mathbf{u}^{T} \mathbf{t} d \mathbf{A} .
\end{aligned}
$$

\subsection{Isogeometric finite element discretization}

For the spatial discretization and solution of the virtual work equation (20) we use the isogeometric finite element method, which was introduced in [24]. In addition to the isoparameteric concept [5, 14], which means that the same function spaces are used for the mathematical description of geometry $\mathbf{X}$ and displacement solution $\mathbf{u}$, the idea behind isogeometric finite elements is to employ the same class of function in the numerical method as already used to define the geometry for example in a CAD program, i.e. B-Splines and NURBS. For a detailled introduction into spline functions we refer to [25] and for a collection of results and applications of isogeometric analysis to [29].

Starting point for the numerical solution of 20 is a trivariate NURBS volume parameterization of material coordinates, i.e. the geometry function mapping a parameter domain $\Omega_{0} \subset \mathbb{R}^{3}$ onto the material coordinates $\mathbf{X} \in \Omega \subset \mathbb{R}^{3}$ :

$\mathbf{X}(\xi)=\sum_{i=1}^{n} N_{i}^{p}(\xi) \mathbf{C}_{i}, \quad \xi \in \Omega_{0}$

Here $n, p, i$ should be understood as 3-dimensional multiindices $n=\left(n_{1}, n_{2}, n_{3}\right), p=\left(p_{1}, p_{2}, p_{3}\right)$ and $i=\left(i_{1}, i_{2}, i_{3}\right)$, giving the number, degree and index of trivariate NURBS functions $N_{i}^{p}(\xi)$ with parameters $\xi=\left(\xi_{1}, \xi_{2}, \xi_{3}\right)$, and $\mathbf{C}_{i} \in$ $\mathbb{R}^{3}$ are the control points of the NURBS volume. The parameter domain $\Omega_{0}$ is also a tensor product of 1-dimensional intervals for each parameter direction, given by knot vectors. Elements in the parameter domain are defined as knot intervals, with the total number of elements denoted by $\ell=$ $\left(\ell_{1}, \ell_{2}, \ell_{3}\right)$.
Following the isoparameteric concept, displacements, velocities and test functions are discretized using the pushforward of NURBS functions onto the material domain:

$$
\begin{aligned}
& \mathbf{u}^{h}(\mathbf{X}, t)=\sum_{i=1}^{n} \hat{N}_{i}^{p}(\mathbf{X}) \mathbf{d}_{i}(t)=\sum_{i=1}^{n} N_{i}^{p}(\xi(\mathbf{X})) \mathbf{d}_{i}(t) \\
& \mathbf{v}^{h}(\mathbf{X}, t)=\sum_{i=1}^{n} \hat{N}_{i}^{p}(\mathbf{X}) \dot{\mathbf{d}}_{i}(t)=\sum_{i=1}^{n} N_{i}^{p}(\xi(\mathbf{X})) \dot{\mathbf{d}}_{i}(t), \\
& \delta \mathbf{u}^{h}(\mathbf{X})=\sum_{i=1}^{n} \hat{N}_{i}^{p}(\mathbf{X}) \delta \mathbf{d}_{i}=\sum_{i=1}^{n} N_{i}^{p}(\xi(\mathbf{X})) \delta \mathbf{d}_{i} .
\end{aligned}
$$

Here $\mathbf{d}_{i}(t) \in \mathbb{R}^{3}$ express the displacements of control points $\mathbf{X}_{i}$ and $\xi(\mathbf{X})$ is the inverse of the geometry mapping (21).

Then the kinematic quantities described in Section 2.1 can be derived in dependence of the discretized displacements from 22. For the deformation gradient this means:

$$
\begin{aligned}
\mathbf{F}(\mathbf{X}, t) & =\mathbf{I}+\nabla \mathbf{u}^{h}(\mathbf{X}, t)=\mathbf{I}+\sum_{i=1}^{n} \mathbf{d}_{i}(t) \nabla \hat{N}_{i}^{p}(\mathbf{X}) \\
& =\mathbf{I}+\sum_{i=1}^{n} \mathbf{d}_{i}(t) \frac{d N_{i}^{p}}{d \xi}(\xi(\mathbf{X})) \cdot\left(\frac{d \mathbf{X}}{d \xi}\right)^{-1} .
\end{aligned}
$$

Cauchy-Green and Green-Lagrange strain tensors can the be computed from $\mathbf{F}$ and the 2nd Piola-Kirchhoff stress be evaluated. Switching to the Voigt vector notation for matrices $\mathbf{E}$ and $\mathbf{S}$ (see [5])

$$
\begin{aligned}
\mathbf{E} & =\left(E_{11}, E_{22}, E_{33}, 2 E_{12}, 2 E_{23}, 2 E_{13}\right)^{T}, \\
\mathbf{S} & =\left(S_{11}, S_{22}, S_{33}, S_{12}, S_{23}, S_{13}\right)^{T}, \\
\mathbf{C}^{S E} & =\frac{d \mathbf{S}}{d \mathbf{E}},
\end{aligned}
$$

the virtual Green-Lagrange strain tensor reads

$\delta \mathbf{E}_{i}=\mathbf{B}_{i}(\mathbf{X}) \delta \mathbf{d}_{i}$,

where the matrix $\mathbf{B}_{i} \in \mathbb{R}^{6 \times 3}$ is

$$
\mathbf{B}_{i}(\mathbf{X}) \hat{=} \frac{1}{2}\left(\nabla \hat{N}_{i}^{p}(\mathbf{X})^{T} \mathbf{F}(\mathbf{X})+\mathbf{F}(\mathbf{X})^{T} \nabla \hat{N}_{i}^{p}(\mathbf{X})\right) .
$$

Then the entries of the internal force vector

$\mathbf{r}_{i}=\sum_{e=1}^{\ell}\left\{\int_{\Omega_{e}} \mathbf{B}_{i}^{T} \mathbf{S} d \mathbf{X}\right\}$

external force vector

$\mathbf{f}_{i}=\sum_{e=1}^{\ell}\left\{\int_{\Omega_{e}} \rho_{0} \hat{N}_{i}^{p} \mathbf{b} d \mathbf{X}+\int_{\Gamma_{n, \ell}} \hat{N}_{i}^{p} \mathbf{t} d \mathbf{A}\right\}$,

and mass matrix

$\mathbf{M}_{i j}=\sum_{e=1}^{\ell}\left\{\mathbf{I} \int_{\Omega_{e}} \rho_{0} \hat{N}_{i}^{p} \hat{N}_{j}^{p} d \mathbf{X}\right\}$, 
can be assembled element-wise on element domains $\Omega_{e}$, just as in standard finite element methods [5, 14], and finally the discretized equation of motion

$\mathbf{M} \ddot{\mathbf{d}}(t)+\mathbf{r}(\mathbf{d}(t))=\mathbf{f}(t)$

needs to be solved for the unknown vector of control point displacements $\mathbf{d}$.

For the solution of 30 we will also need the tangential stiffness matrix

$\mathbf{K}_{T}=\frac{d \mathbf{r}}{d \mathbf{d}}=\mathbf{K}^{g e o}+\mathbf{K}^{m a t}$,

with the assembled geometric tangent matrix:

$\mathbf{K}_{i j}^{g e o}=\sum_{e=1}^{\ell}\left\{\mathbf{I} \int_{\Omega_{e}} \nabla \hat{N}_{i}^{p T} \mathbf{S} \nabla \hat{N}_{j}^{p} d \mathbf{X}\right\}$,

and material tangent matrix:

$\mathbf{K}_{i j}^{m a t}=\sum_{e=1}^{\ell}\left\{\int_{\Omega_{e}} \mathbf{B}_{i}^{T} \mathbf{C}^{S E} \mathbf{B}_{j} d \mathbf{X}\right\}$

\section{Nonlinear analysis of structural vibrations}

Having introduced the problem formulation of nonlinear structural dynamics and the spatial discretization using isogeometric finite elements in the previous Section 2, we now target the topic of frequency analysis. Therefore we start with a brief review of methods for linear frequency analysis, i.e. modal analysis of eigenfrequencies and eigenforms, and direct frequency response to harmonic excitations in Section 3.1 Then we introduce the method of harmonic balance, which allows to compute nonlinear steady-state frequency response to periodic excitations, in Section 3.2

\subsection{Linear frequency analysis}

In linear elasticity strains are restricted to small deformation theory, compare (5),

$\mathbf{e}(\mathbf{X}, t)=\frac{1}{2}\left(\nabla \mathbf{u}^{T}+\nabla \mathbf{u}\right)$,

and for the constitutive relation the linear St. Venant-Kirchhoff law is used, see 9],

$\sigma=\lambda \operatorname{tr}(\mathbf{e}) \mathbf{I}+2 \mu \mathbf{e}$.

The (isogeometric) finite element discretization analogous to Section 2.3 leads to the following semi-discretized, time-dependent problem of linear elasto-dynamics [14,29]:

$\mathbf{M} \ddot{\mathbf{d}}(t)+\mathbf{K} \mathbf{d}(t)=\mathbf{f}(t) \quad \forall t \in[0, T]$,

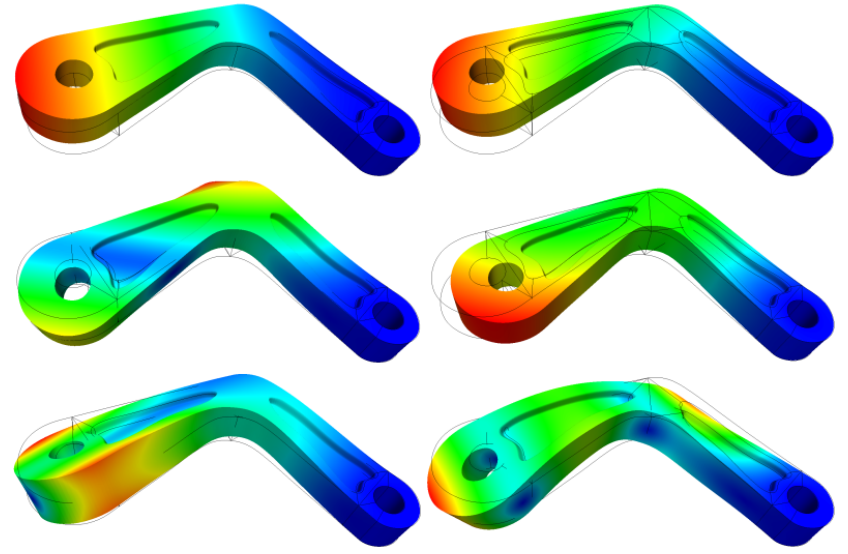

Fig. 2: First six eigenmodes of the so-called TERRIFIC demonstrator, computed with IGA from a multi-patch model with 15 blocks

where $\mathbf{M}$ and $\mathbf{K}$ are the $n \times n$ mass and linear stiffness matrix, $\mathbf{d}$ is the vector of control point displacements and $\mathbf{f}$ is the vector of external forces. Furthermore we have periodicy conditions for the displacement vectors:

$\mathbf{d}(0)=\mathbf{d}(T), \quad \mathbf{v}(0)=\mathbf{v}(T)$.

\subsubsection{Modal analysis of eigenfrequencies}

From (36) one can derive the well-known eigenvalue problem

$-\omega_{k}^{2} \mathbf{M} \phi_{k}+\mathbf{K} \phi_{k}=\mathbf{0}, k=1, \ldots, n$,

for the $n$ linear natural frequencies $\omega_{k}$ and corresponding eigenmodes $\phi_{k}[14,29]$.

In [30] the properties of isogeometric finite element discretizations in the context of linear eigenvalue problems such as (38) were examined already. For one-dimensional rods and beams, it has been shown analytically and numerically that spline-based finite elements are more accurate than Lagrangian finite elements. While $C^{0}$-continuous Lagrangian FE of higher degrees $p>1$ exhibit optical and acoustical branches in the frequency spectrum with huge errors and no $p$-convergence in higher eigenfrequencies, $C^{p-1}$-continuous spline-based FE shows high accuracy and $p$-convergence over the whole spectrum. These results have also been numerically verified in [30] for 2- and 3-dimensional linear eigenvalue problems and thus motivate the use of isogeometric finite elements in vibration analysis. Furthermore the dependency of convergence of IGA on the type of parameterization - linear or uniform - was investigated in [35].

An example for modal analysis using isogeometric finite elements is shown in Figure 2 with the TERRIFIC demonstrator part [36], which consists of 15 NURBS patches. This application is going to be addressed in more detail in Section 5.3 . 


\subsubsection{Direct frequency response}

Another means of linear frequency analysis, which is also available in most FEA software, is the so-called direct frequency response (DFR) method. Given a harmonic external loading of the form

$\mathbf{f}(t)=\mathbf{f}_{c} \cos \omega t+\mathbf{f}_{s} \sin \omega t$,

the steady-state reponse of the structure is assumed as

$\mathbf{d}(t)=\mathbf{q}_{c} \cos \omega t+\mathbf{q}_{s} \sin \omega t$

A transformation of (36), including an additional damping term $\mathbf{C} \dot{\mathbf{d}}(t)$, onto the Fourier domain then yields a linear system of equations for the unknown cosine and sine amplitudes of the displacement $\mathbf{q}_{c}$ and $\mathbf{q}_{c}$ :

$-\omega^{2} \mathbf{M} \mathbf{q}_{c}+\omega \mathbf{C} \mathbf{q}_{s}+\mathbf{K} \mathbf{q}_{c}=\mathbf{f}_{c}$,

$-\omega^{2} \mathbf{M} \mathbf{q}_{s}-\omega \mathbf{C} \mathbf{q}_{c}+\mathbf{K} \mathbf{q}_{s}=\mathbf{f}_{s}$.

3.2 Nonlinear frequency response: the Harmonic Balance Method

While the solution of eigenvalue problems and direct frequency response are two standard methods in engineering practise for the frequency and vibration analysis of structures, nonlinear vibration analysis is a much harder task which is lacking efficient numerical methods. Steady-state vibration response of a structure subject to nonlinearities such as large deformations and hyperelastic material models (see Section 2.1) is typically solved by time-integration of finite element models, also in the IGA context [5, 29].

A more elegant approach is the Harmonic Balance Method (HBM) [1-3], which has been employed so far mainly for FE-discretizations of beam and shell models only [9. 13]. Furthermore we have already investigated the HBM in conjunction with IGA for nonlinear Euler-Bernoulli beams [4]. We could show that IGA is more accurate than standard or $p$-FEM in this setting as well, due to the higher $C^{p-1}$. continuity.

Here we apply the method to our isogeometric finite element discretization of large deformation hyperelasticity (see Section 2.3. Therefore we start from the equation of motion (30) with periodicy conditions:

$$
\begin{gathered}
\mathbf{M} \ddot{\mathbf{d}}(t)+\mathbf{r}(\mathbf{d}, t)=\mathbf{f}(t) \quad \forall t \in[0, T], \\
\mathbf{d}(0)=\mathbf{d}(T), \quad \mathbf{v}(0)=\mathbf{v}(T) .
\end{gathered}
$$

Now we consider only periodic external excitations of the structure, with frequency $\omega$ (period $T=2 \pi / \omega)$ and a finite number $m^{*}$ of higher harmonics:

$\mathbf{f}(\omega, t)=\frac{1}{2} \mathbf{f}_{0}+\sum_{k=1}^{m^{*}} \cos (k \omega t) \mathbf{f}_{k}+\sin (k \omega t) \mathbf{f}_{2 m^{*}-k+1}$.
We expect the response to periodic excitation to be $\omega$-periodic as well and therefore express the displacement coefficients $\mathbf{d}(t)$ of the spatial discretization $\mathbf{u}^{h}(\mathbf{X}, t)$ 22) (and consequently also the velocities and accelerations) as a truncated Fourier expansion with $m \geq m^{*}$ harmonic terms of frequency $\omega$ and amplitudes $\mathbf{q}=\left(\mathbf{q}_{0}, \ldots, \mathbf{q}_{2 m}\right)$ :

$\mathbf{d}(\mathbf{q}, \omega, t)=\frac{1}{2} \mathbf{q}_{0}+\sum_{k=1}^{m} \cos (k \omega t) \mathbf{q}_{k}+\sin (k \omega t) \mathbf{q}_{\bar{k}}$,

$\dot{\mathbf{d}}(\mathbf{q}, \omega, t)=\sum_{k=1}^{m}-k \omega \sin (k \omega t) \mathbf{q}_{k}+k \omega \cos (k \omega t) \mathbf{q}_{\bar{k}}$,

$\ddot{\mathbf{d}}(\mathbf{q}, \omega, t)=\sum_{k=1}^{m}-k^{2} \omega^{2} \cos (k \omega t) \mathbf{q}_{k}-k^{2} \omega^{2} \sin (k \omega t) \mathbf{q}_{\bar{k}}$,

with the abbreviation $\bar{k}=2 m-k+1$.

When we substitue the ansatz from (44) into (42), we get a resiudal vector:

$\varepsilon(\mathbf{q}, \omega, t)=\mathbf{M} \ddot{\mathbf{d}}(\mathbf{q}, \omega, t)+\mathbf{r}(\mathbf{q}, \omega, t)-\mathbf{f}(\omega, t)$.

In the next step we apply the Ritz procedure by projecting the residual $\varepsilon$ onto the temporal basis functions, in order to obtain a Fourier expansion of the residual with $2 m+1$ coefficient vectors that have to be evaluated to $\mathbf{0}$ (balance of the harmonics):

$\varepsilon_{j}(\mathbf{q}, \omega)=\frac{2}{T} \int_{0}^{T} \varepsilon(\mathbf{q}, \omega, t) \cos j \omega t d t \stackrel{!}{=} \mathbf{0}, j=0, \ldots, m$,

$\varepsilon_{\bar{j}}(\mathbf{q}, \omega)=\frac{2}{T} \int_{0}^{T} \varepsilon(\mathbf{q}, \omega, t) \sin j \omega t d t \stackrel{!}{=} \mathbf{0}, j=1, \ldots, m$.

The nonlinear system of $(2 m+1) \cdot n$ equations given by needs to be solved in order to determine the amplitudes $\mathbf{q}$ for given $\omega$.

Note that the existence and accuracy of a solution may highly depend on the number of harmonics $m$, since nonlinear effects, such as internal resonance and coupling of modes, typically cause response in higher harmonics $m>m^{*}$ than the highest excited harmonic.

For computational purposes the abovementioned equations may be transformed onto a non-dimensional time $\tau=$ $\omega t$. Then the domain of integrals in (46) becomes $[0,2 \pi]$ and for computation we can use discrete Fourier resp. Hartley transform (DFT, DHT), where the residual $\varepsilon(\mathbf{q}, \omega, \tau)$ has to be sampled at $2 m+1$ equidistant times $\tau_{j}=\frac{2 \pi j}{2 m+1}, j=$ $0, \ldots, 2 m$.

Furthermore, for solving (46) with a Newton's method we need the Jacobians of residual coefficients $\varepsilon_{j}$ with respect to amplitudes $\mathbf{q}_{k}, k=0, \ldots, 2 m$ :

$\frac{d \varepsilon_{j}}{d \mathbf{q}_{k}}(\mathbf{q}, \omega)=\frac{2}{T} \int_{0}^{T} \frac{d \varepsilon}{d \mathbf{q}_{k}}(\mathbf{q}, \omega, t) \cos j \omega t d t, j=0, \ldots, m$,
$\frac{d \varepsilon_{\bar{j}}}{d \mathbf{q}_{k}}(\mathbf{q}, \omega)=\frac{2}{T} \int_{0}^{T} \frac{d \varepsilon}{d \mathbf{q}_{k}}(\mathbf{q}, \omega, t) \sin j \omega t d t, j=1, \ldots, m$. 
The integrals are again evaluated by discrete Fourier transform and thereby we also need the Jacobians of the residual $\varepsilon$ with respect to amplitudes $\mathbf{q}_{k}$ :

$$
\begin{aligned}
\frac{d \varepsilon}{d \mathbf{q}_{k}}(\mathbf{q}, \omega, t) & =-k^{2} \omega^{2} \cos k \omega t \mathbf{M} \\
& +\cos k \omega t \mathbf{K}_{T}(\mathbf{q}, \omega, t), k=0, \ldots, m \\
\frac{d \varepsilon}{d \mathbf{q}_{\bar{k}}}(\mathbf{q}, \omega, t) & =-k^{2} \omega^{2} \sin k \omega t \mathbf{M} \\
& +\sin k \omega t \mathbf{K}_{T}(\mathbf{q}, \omega, t), k=1, \ldots, m
\end{aligned}
$$

with $\mathbf{K}_{T}=\frac{d \mathbf{r}}{d \mathbf{d}}$ from 31 .

Response curves (RC) are a wide-spread means of visualizing the frequency response of steady-state vibrating systems, also for linear DFR. Typically, the total amplitude $A_{k}$ and phase $\phi_{k}$ of one or more harmonics $k$ are evaluated at a specific point on the structure for fixed $\omega$ and plotted over a certain range of frequency. They can be computed as follows:

$$
\begin{aligned}
& A_{k}=\sqrt{q_{k}^{2}+q_{\bar{k}}^{2}}, \quad \phi_{k}=\arctan \frac{q_{\bar{k}}}{q_{k}}, \\
& q_{k} \cos (k \omega t)+q_{\bar{k}} \sin (k \omega t)=A_{k} \cos \left(k \omega t+\phi_{k}\right) .
\end{aligned}
$$

The simplest method for generating response curves and functions is simply to start from a fixed $\omega$, compute the response via HBM or DFR, evaluate amplitude and phase at the evaluation point, and then increment the frequency step by step. However, for complex vibrational behaviour, bifurcations and turning points might occur and make it necessary to use arc-length continuation methods, which we have not done for our numerical examples presented in Section 5 .

\section{Model order reduction for nonlinear vibration analysis}

As mentioned in Section 3.2, the harmonic balance method for nonlinear vibration analysis requires the solution of a linear system of equations of size $n \cdot(2 m+1)$ in each step of a Newton iteration, where $n$ is the number of spatial DOFs and $m$ the number of harmonics in the Fourier expansion of each DOF. This system is not only $(2 m+1)$-times bigger than the underlying static system (i.e. the tangent stiffness matrix $K_{T}$ ), but also much more densely populated. Each row of $\frac{d \varepsilon_{j}}{d \mathbf{q}_{k}}$ also has $(2 m+1)$-times the number of non-zero entries of the corresponding row of $K_{T}$ and is not symmetric anymore.

This is a severe draw-back regarding the solution process of the system using sparse linear solvers, which are designed for the solution of large systems with only few non-zero entries per row. While sampling time for Fourier transform, i.e.
$(2 m+1)$-times assembly of force vector and tangent stiffness, increases linearily with $m$, and time for Fourier transform itself by $m \log m$, solution time of the system increases more rapidly. For complex engineering structures with hundreds of thousands or even millions of DOFs in the finite element model, a harmonic balance analysis becomes even impossible.

Thus we are looking for a suitable model order reduction method (MOR) in order to decrease the computational effort for solving the linear system within harmonic balance and allowing a nonlinear frequency analysis even for large-scale applications.

\subsection{Overview of model order reduction methods}

Having identified the need for model order reduction, we are giving a brief review of different kinds of model reduction methods with applications in nonlinear structural mechanics and dynamics [5, 14, 15].

There is a wide range of projection based reduction methods, where the physical coordinate vector $\mathbf{d} \in \mathbb{R}^{n}$ can be expressed by a linear transformation of reduced coordinates $\hat{\mathbf{d}} \in \mathbb{R}^{r}$ :

$\mathbf{d}=\Phi \hat{\mathbf{d}}$.

$\Phi \in \mathbb{R}^{n \times r}$ is the transformation matrix, with $\operatorname{rank}(\Phi)=r \leq$ $n$. In case of $r=n$ (51) is a basis transformation, but the intention is to chose $r \ll n$ and project onto a smaller subspace of the original solution space.

The most common projection method is modal reduction or truncation [14,15], where the transformation matrix $\Phi$ is composed from a subset of linear eigenvectors, see (38). It is widely used in linear structural dynamics, but there is only a limited applicability in nonlinear analysis (see also later examples in Section 5.1).

Tangent modes [21,22] are the eigenmodes one obtains from the solution of an updated eigenvalue problem with the tangent stiffness $\mathbf{K}_{T}$ for the current deformation state. In nonlinear time-integration an updated modal basis can be determined from these tangent modes in every time step, or after a suitable number of time steps. In [21] also a method for direct update of eigenvectors in each time step is described. But as we compute the amplitudes for one whole period of vibration in harmonic balance, there is no specific current state of deformation in our setting and these methods seem not very applicable.

A nonlinear counterpart of linear eigenmodes are nonlinear normal modes (NNMs), which have shown good results in nonlinear frequency analysis in terms of self-excited vibrations before [16, 37,-39]. However, the computational effort of numerically determining the NNMs seems very high, since for example one method is solving an autonomous harmonic balance problem for the full system for each mode. 
Another means of generating a projection basis are Ritz vectors [21,22]. Ritz vectors are developed from load or displacement of a current state; for nonlinear analysis basis updates and derivatives may also be included, providing a good approximation of exact solutions [21]. However, again this method relies on a fixed current state of deformation and load and seems not suitable for application in harmonic balance.

For Proper Orthogonal Decomposition (POD) a set of sample displacement vectors has to be generated as part of preprocessing, from which an optimal basis is created [21. 40,41]. The method leads to good results in structural time integration, but sampling requires a priori knowledge of loads Since unexpected resonances and states of deformation are to be found in nonlinear vibration analysis with HBM, the POD method might not be suitable for those.

Our choice of reduction method is modal reduction resp. truncation (MR) with modal derivatives [17- 22], which we present in detail in the next Section 4.2. Modal derivatives (MD) are a second order enhancement of the modal basis, which accounts for quadratic terms as they appear in large deformation theory and Green-Lagrange strains. The modal derivatives can be computed from the linear stiffness matrix and eigenvectors as part of preprocessing and do not require basis updates during the computation.

\subsection{Modal reduction with modal derivatives}

As it is our method of choice for the use in nonlinear vibration analysis, we give an introduction into the concept of modal derivatives and their computation, using references [17, 18, 20]:

In a linear modal truncation the displacements are expressed in terms of eigenmodes of the linear problem $\phi_{i}$ and modal coordinates $\hat{d}_{i}$. Since the tangent stiffness matrix depends on displacements in a nonlinear setting, also the (tangent) modes depend on displacements:

$\mathbf{d}=\sum_{i=1}^{n} \phi_{i}(\mathbf{d}) \hat{d}_{i}$

Now $\mathbf{d}$ is developed as second-order Taylor series around the initial configuration of zero displacements $\mathbf{d}=\mathbf{0}(\hat{\mathbf{d}}=\mathbf{0})$ :

$$
\begin{aligned}
\mathbf{d} & =\mathbf{0}+\sum_{i=1}^{n}\left(\frac{\partial \mathbf{d}}{\partial \hat{d}_{i}}(\hat{\mathbf{d}}=\mathbf{0}) \hat{d}_{i}+\sum_{j=1}^{n} \frac{\partial^{2} \mathbf{d}}{\partial \hat{d}_{i} \partial \hat{d}_{j}}(\hat{\mathbf{d}}=\mathbf{0}) \frac{\hat{d}_{i} \hat{d}_{j}}{2}\right) \\
& =\sum_{i=1}^{n}\left(\phi_{i}(\mathbf{0}) \hat{d}_{i}+\sum_{j=1}^{n}\left(\frac{\partial \phi_{j}}{\partial \hat{d}_{i}}(\mathbf{0})+\frac{\partial \phi_{i}}{\partial \hat{d}_{j}}(\mathbf{0})\right) \frac{\hat{d}_{i} \hat{d}_{j}}{2}\right) .
\end{aligned}
$$

For computing the modal derivatives $\frac{\partial \phi_{j}}{\partial \hat{d}_{i}}$ one needs to differentiate the eigenvalue problem (38) with $\mathbf{K}=\mathbf{K}_{T}(\mathbf{0})$ w.r.t. to the modal coordinates:

$$
\begin{aligned}
\frac{\partial}{\partial \hat{d}_{i}} & {\left[\left(\mathbf{K}-\omega_{j}^{2} \mathbf{M}\right) \phi_{j}\right]=} \\
& \left(\mathbf{K}-\omega_{j}^{2} \mathbf{M}\right) \frac{\partial \phi_{j}}{\partial \hat{d}_{i}}+\left(\frac{\partial \mathbf{K}}{\partial \hat{d}_{i}}-\frac{\partial \omega_{j}^{2}}{\partial \hat{d}_{i}} \mathbf{M}\right) \phi_{j}=\mathbf{0} .
\end{aligned}
$$

In [18] three different approaches for the solution of (54) are presented: analytical, analytical excluding inertia effects and purely numerical using finite differences of the re-computed tangent eigenvalue problem. We have implemented the enhanced modal basis approach using modal derivatives with the "analytical approach excluding mass consideration", which leads to the solution of the following linear system for $\frac{\partial \phi_{j}}{\partial \hat{d}_{i}}$ :

$\frac{\partial \phi_{j}}{\partial \hat{d}_{i}}=-\mathbf{K}^{-1} \frac{\partial \mathbf{K}}{\partial \hat{d}_{i}} \phi_{j}$,

with a finite difference approximation of the derivative of the tangent stiffness matrix

$\frac{\partial \mathbf{K}}{\partial \hat{d}_{i}} \simeq \frac{\mathbf{K}_{T}\left(\Delta \hat{d}_{i} \phi_{i}\right)-\mathbf{K}}{\Delta \hat{d}_{i}}$.

Thus we can compute approximations symmetric modal derivatives $\frac{\partial \phi_{j}}{\partial \hat{d}_{i}}$, which we also ortho-normalize. An extended reduction basis with $r_{d}$ eigenmodes and the corresponding modal derivatives is:

$\Phi=\left(\phi_{1}, \ldots, \phi_{r_{d}}, \frac{\partial \phi_{1}}{\partial \hat{d}_{1}}, \frac{\partial \phi_{1}}{\partial \hat{d}_{2}}, \ldots, \frac{\partial \phi_{1}}{\partial \hat{d}_{r_{d}}}, \ldots, \frac{\partial \phi_{r_{d}}}{\partial \hat{d}_{r_{d}}}\right)$,

which then has basis length $r=r_{d}+r_{d}\left(r_{d}+1\right) / 2$ and the linear projection for reduction is:

$\mathbf{d}=\Phi \hat{\mathbf{d}}=\sum_{i=1}^{r_{d}} \phi_{i} \hat{d}_{i}+\sum_{i=1}^{r_{d}} \sum_{j=i}^{r_{d}} \frac{\partial \phi_{i}}{\partial \hat{d}_{j}} \hat{d}_{i j}$

Note that from the quadratic Taylor expansion in 53 with dependent quadratic coefficients $\hat{d}_{i} \hat{d}_{j}$ we have generated a reduced linear expansion in (58) with independent coefficients $\hat{d}_{i j}$.

Currently there are no theoretical error estimates available for the accuracy of reduction with the modal derivative approach and the number and choice of modes and derivatives to be included in the basis (57) has to be manually selected. However, the numerical results presented in Section 5. especially the static convergence study in Section 5.1, indicate convergence with respect to the number of basis vectors used and acceptable accuracy already for a small number of modes and derivatives.

Remark that the computational effort for computing all $r_{d}\left(r_{d}+1\right) / 2$ symmetric modal derivatives is mainly computing the $r_{d}$ linear eigenvectors $\phi_{j}$, assembly of $r_{d}$-times a tangent stiffness matrix in 56 and then solving the linear system 55) $r_{d}\left(r_{d}+1\right) / 2$-times. If the problem size is not too big and one can solve (55) by LU-decomposition of $\mathbf{K}$, the computation becomes very efficient! 
4.3 Application of reduction to nonlinear vibration analysis

Here we describe the application of the modal projection method with eigenmodes and modal derivatives, as introduced in the preceding Section 4.2 to the harmonic balance method, see Section 3.2 .

The displacement vector $\mathbf{d}$ in the equation of motion of harmonic balance (42) is projected using modal coordinates $\hat{\mathbf{d}}$ :

$\mathbf{d}(t)=\Phi \hat{\mathbf{d}}(t)$,

where $\Phi$ is the projection matrix with a selection of $r \ll n$ linear eigenmodes $\phi_{i}$ and corresponding modal derivatives $\frac{\partial \phi_{j}}{\partial \hat{d}_{i}}$ as columns, see 58. .

Now the equation of motion (42) is transformed onto modal coordinates by left-multiplication with $\Phi^{T}$ :

$\Phi^{T} \mathbf{M} \Phi \ddot{\hat{\mathbf{d}}}(t)+\Phi^{T} \mathbf{r}(\mathbf{d}(t))=\Phi^{T} \mathbf{f}(t)$

This equation may be re-written using the notations

$\hat{\mathbf{M}}=\Phi^{T} \mathbf{M} \Phi, \hat{\mathbf{r}}=\Phi^{T} \mathbf{r}, \hat{\mathbf{f}}=\Phi^{T} \mathbf{f}$

as

$\hat{\mathbf{M}} \ddot{\hat{\mathbf{d}}}(t)+\hat{\mathbf{r}}(\mathbf{d}(t))=\hat{\mathbf{f}}(t)$.

The spatial discretization of the nonlinear equation system has been reduced from a sparse $n \times n$ to a dense $r \times r$ one, but note that the nonlinear term $\hat{\mathbf{r}}(\mathbf{d}(t))$ (and also the corresponding tangent $\left.\hat{\mathbf{K}}_{T}(\mathbf{d}(t))=\Phi^{T} \mathbf{K}_{T}(\mathbf{d}(t)) \Phi\right)$ still depend on the physical displacement vector $\mathbf{d}$ and have to be assembled in the usual way without any speed-up. Therefore dimension reduction methods such as Discrete Empirical Interpolation [42] might be applied in the future.

The further procedure of applying the harmonic balance method is equivalent to the steps described in Section 3.2. Fourier expansion of modal coordinates $\hat{\mathbf{d}}$ using amplitudes $\hat{\mathbf{q}}$, substitution into (62) for a resdiual vector $\hat{\varepsilon}$, Fourier transform of the residual and its Jacobian, and solution of the equation system for $\hat{\mathbf{q}}$, which has now size $r \cdot(2 m+1)$. Physical displacements can then be recovered within each sampling or Newton step using $\mathbf{d}(t)=\Phi \hat{\mathbf{d}}(t)$.

A schematic overview of the algorithm for nonlinear frequency analysis with modal derivative reduction is presented in Figure 3 .

\section{Computational applications and examples}

5.1 Modal reduction in 3D nonlinear elasto-statics

Before applying the modal reduction technique from Section 4.2 to nonlinear vibration analysis, we want to study the
Compute reduction basis (pre-processing):

Assemble $\mathbf{M}, \mathbf{K}$

Solve $-\omega_{j}^{2} \mathbf{M} \phi_{j}+\mathbf{K} \phi_{j}=\mathbf{0}, j=1, \ldots, r$

For $i=1, \ldots, r$ :

Assemble $\mathbf{K}_{T}\left(\Delta \hat{d}_{i} \phi_{i}\right)$

Compute $\frac{\partial \mathbf{K}}{\partial \hat{d}_{i}} \simeq \frac{\mathbf{K}_{T}\left(\Delta \hat{d}_{i} \phi_{i}\right)-\mathbf{K}}{\Delta \hat{d}_{i}}$

For $j=1, \ldots, r$ :

Solve $\frac{\partial \phi_{j}}{\partial \hat{d}_{i}}=-\mathbf{K}^{-1} \frac{\partial \mathbf{K}}{\partial \hat{d}_{i}} \phi_{j}$

Basis $\Phi=\left(\phi_{1}, \ldots, \phi_{r_{d}}, \frac{\partial \phi_{1}}{\partial \hat{d}_{1}}, \ldots, \frac{\partial \phi_{1}}{\partial \hat{d}_{r_{d}}}, \ldots, \frac{\partial \phi_{r_{d}}}{\partial \hat{d}_{r_{d}}}\right)$

Frequency response for $\omega$ :

While $\|\hat{\varepsilon}\|>0$ (Newton iteration):

For $j=0, \ldots, 2 m$ (sampling):

Displacement $\mathbf{d}\left(\tau_{j}\right)=\Phi\left(\frac{1}{2} \hat{\mathbf{q}}_{0}+\sum_{k=1}^{m} \cos \left(k \tau_{j}\right) \hat{\mathbf{q}}_{k}\right.$ $\left.+\sin \left(k \tau_{j}\right) \hat{\mathbf{q}}_{\bar{k}}\right)$

Assemble $\mathbf{r}\left(\tau_{j}\right), \mathbf{K}_{T}\left(\tau_{j}\right)$

Evaluate $\varepsilon\left(\tau_{j}\right), \frac{d \varepsilon}{d \mathbf{q}_{k}}\left(\tau_{j}\right)$

Reduction $\hat{\varepsilon}\left(\tau_{j}\right)=\Phi^{T} \varepsilon\left(\tau_{j}\right)$,

$$
\frac{d \hat{\varepsilon}}{d \hat{\mathbf{q}}_{k}}\left(\tau_{j}\right)=\Phi^{T} \frac{d \varepsilon}{d \mathbf{q}_{k}}\left(\tau_{j}\right) \Phi
$$

Fourier transform $\hat{\varepsilon}\left(\tau_{j}\right) \rightarrow \hat{\varepsilon}_{j}$,

$$
\begin{aligned}
& \qquad \frac{d \hat{\varepsilon}}{d \hat{\mathbf{q}}_{k}}\left(\tau_{j}\right) \rightarrow \frac{d \hat{\varepsilon}_{j}}{d \hat{\mathbf{q}}_{k}} \\
& \text { Solve } \Delta \hat{\mathbf{q}}=-\left(\frac{d \hat{\varepsilon}}{d \hat{\mathbf{q}}}\right)^{-1} \hat{\varepsilon} \\
& \text { Update } \hat{\mathbf{q}} \leftarrow \hat{\mathbf{q}}+\Delta \hat{\mathbf{q}}
\end{aligned}
$$

Fig. 3: Algorithm for nonlinear frequency analysis with modal derivative reduction

accuracy of modal reduction with modal derivatives for nonlinear large deformation hyperelasticity in a static 3-dimensional case, i.e. solving

$$
\Phi^{T} \mathbf{r}(\mathbf{d})=\Phi^{T} \mathbf{f}
$$

$\mathbf{d}=\Phi \hat{\mathbf{d}}, \quad \Phi \in \mathbb{R}^{n \times r}$.

We use a 3D geometry with no symmetries subject to large deformations and linear St. Venant-Kirchhoff material law. One boundary face of the object is fixed by coupled Dirichlet boundary conditions and then eigenvalues and eigenvectors are computed for setting up the modal matrix $\Phi$ with first $r$ eigenmodes as columns. For reduction with modal derivatives we compute the modal derivative basis with $r_{d}$ eigenmodes and corresponding modal derivatives. For static computations a Neumann boundary condition is applied to the opposite face with force contributions in all 3 dimensions, forcing a large deformation up to $100 \%$ of the object's dimensions. The geometry of the object together with results of subsequent computations is visualized in Figure 4. 

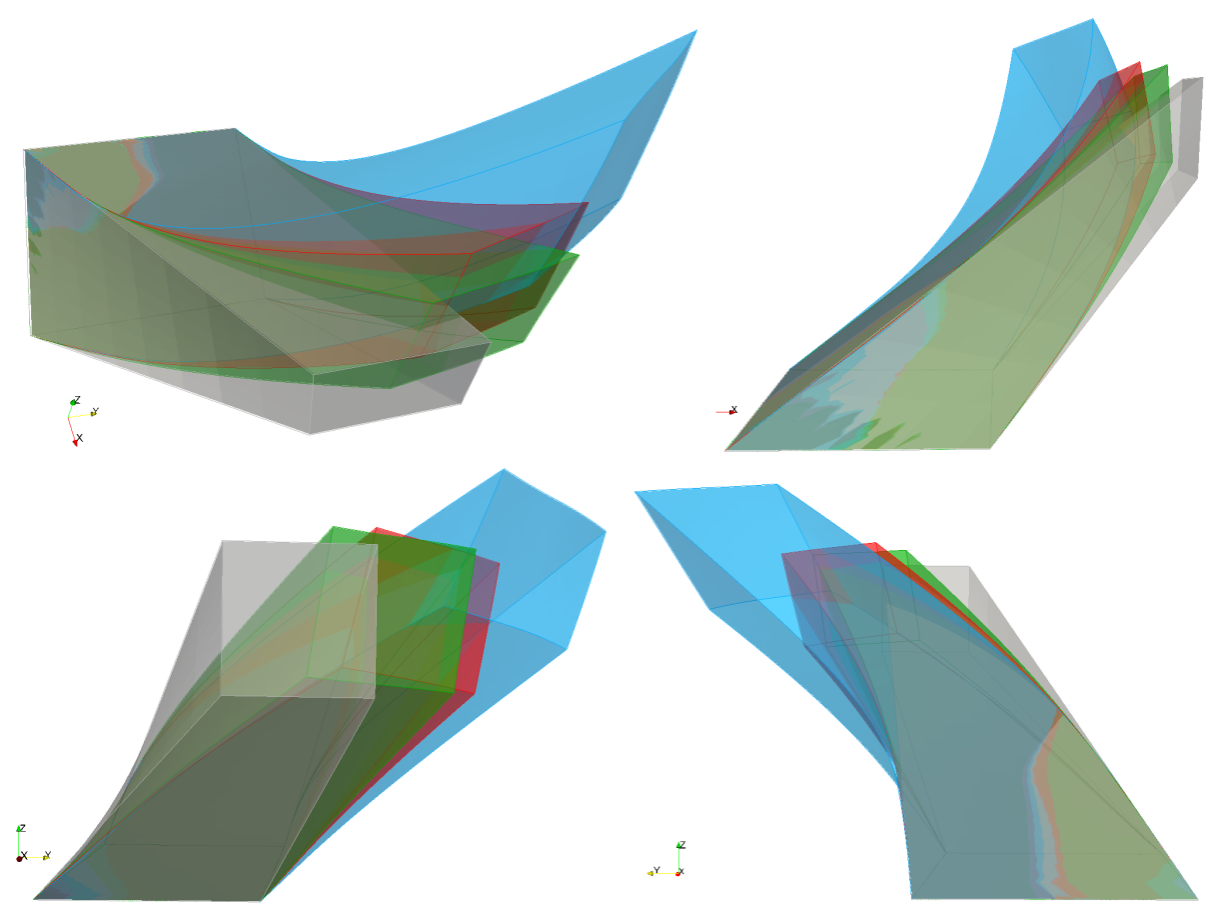

Fig. 4 Geometry of the 3D object (grey), linear displacement (blue), nonlinear displacement (red), nonlinear displacement with modal reduction $r=50$ (green).

For a quite coarse isogeometric discretization with $p=$ $(2,2,2), \ell=(2,2,4), n=(4,4,6), N=288$, we compare the accuracy of modal reduction (MR) and modal reduction with derivatives (MD) with the full, unreduced nonlinear computation. As criteria we use the relative errors of $x$-, $y$ - and $z$-displacement, evaluated on the center point of the surface where the load is applied, e.g. $\left|u_{x}^{\text {full }}-u_{x}^{\mathrm{MD}}\right| /\left|u_{x}^{\mathrm{full}}\right|$, as well as relative errors in $L^{2}$ - and $H^{1}$-norms, e.g. $\| u^{\text {full }}$ $u^{\mathrm{MD}}\left\|_{L^{2}} /\right\| u^{\text {full }} \|_{L^{2}}$.

In Figure 5 we compare the relative $L^{2}$ - and $H^{1}$-errors of the full and reduced solutions for the linear case with MR and the nonlinear case with MR and MD. While no significant improvement of accuracy with increasing basis length (number of modes) is noticable for MR in the nonlinear case, MD provides a similar convergence behaviour as MR in the linear case. Note that for $r=240$ we are already considering the full set of displacement modes and for $r=N$ the transformation is bijective and thus must reproduce the results of the full system.

Furthermore we have also investigated the behaviour of modal reduction and modal derivatives for different load factors. Figure 6 shows the displacement at the evaluation point for load facor 1 to 1000 (100 corresponds to the load level of previous results). While there is no visible deviation from full results for MD, MR shows large errors.

In Figure 7 the relative errors of the displacment at evaluation point and relative $L^{2}$ - and $H^{1}$-norm errors are shown over increasing load factor. While errors for MD are small and roughly stay constant up to very large load factors and thus displacements, relative errors for MR grow fast and to a very high, unreliable level.

With this numerical study we have examined the approximation properties of our modal reduction method for large deformations in a static setting. We can conclude that modal reduction is unsuitable for reduction of large deformation problems, while modal reduction with modal derivatives provides a high accuracy in the nonlinear static problem setting and opens a perspective for the use in nonlinear vibration analysis.

\subsection{Large amplitude vibration of a thick cylinder}

Mathisen et al. already studied the use of isogeometric analysis in compressible and incompressible hyperelasticity with Neo-Hooke materials [34]. We pick up the example, with a geometry that can be exactly represent using a NURBS volume, with a compressible Neo-Hooke material law for nonlinear vibration analysis. The dimensions of on eight of the cylinder, material parameters and loads can be found in Figure 8. The surface Neumann loads are periodic.

For the isogeometric discretization we chose $p=(3,3,3)$, $\ell=(4,4,1), n=(7,7,4), N=588$, and therefore compute the first linear eigenfrequency as $f_{1}^{h}=1581.9 \mathrm{~Hz}$.

Now we perform a harmonic balance frequency response analysis with $m=3$ (HBM) near the first eigenfrequency within the frequency range of $0.85<f / f_{1}^{h}<1.15$ and compare the results with linear direct frequency reponse (DFR). A snapshot of the deformed vibrating cylinder for $f / f_{1}^{h}=$ 0.95 can be seen in Figure 9 . Although very large deforma- 

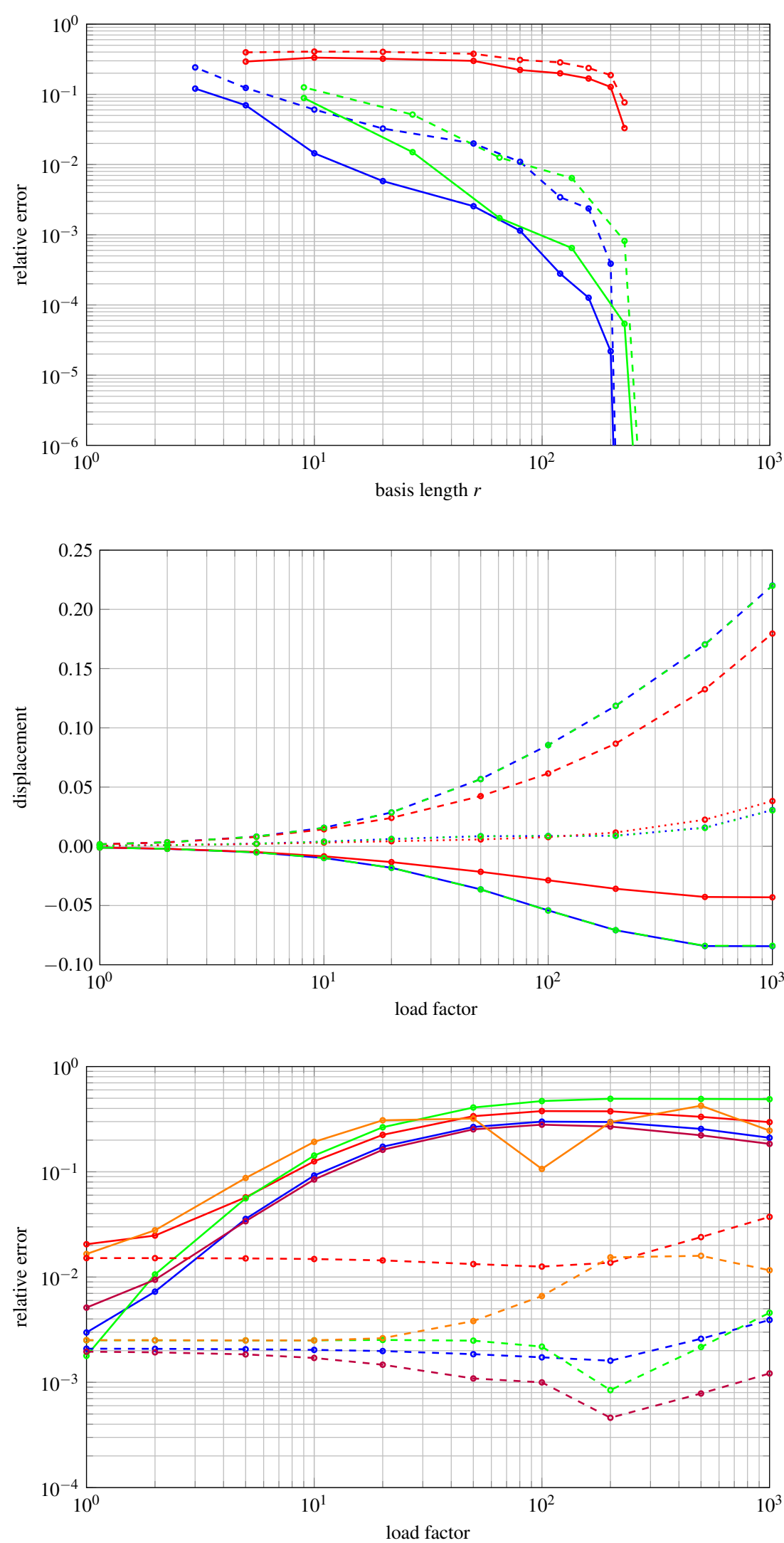

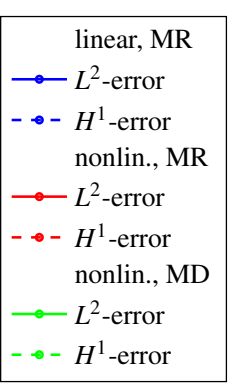

Fig. 5 Convergence of modal reduction w.r.t. basis length $r$ in relative $L^{2}$ - and $H^{1}$-norms. Poor results for modal reduction (MR) in the nonlinear case, while enhanced basis (MD) for nonlinear problem performs as good as modal basis in linear case

\begin{tabular}{|c|}
\hline 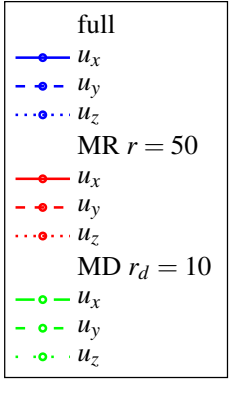 \\
\hline
\end{tabular}

Fig. 6 Displacement at evaluation point for full computation (full), modal reduction with 50 modes (MR $r=50$ ) and modal derivatives for 10 modes (MD $\left.r_{d}=10\right)$ for increasing load factor

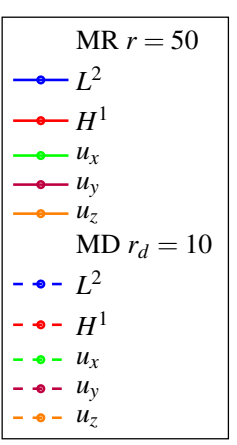

Fig. 7 Relative error of displacement amplitudes, $L^{2}$ and $H^{1}$-norm of MR $(r=$ 50) and $\mathrm{MD}\left(r_{d}=10\right)$ w.r.t full computation for increasing load factor 


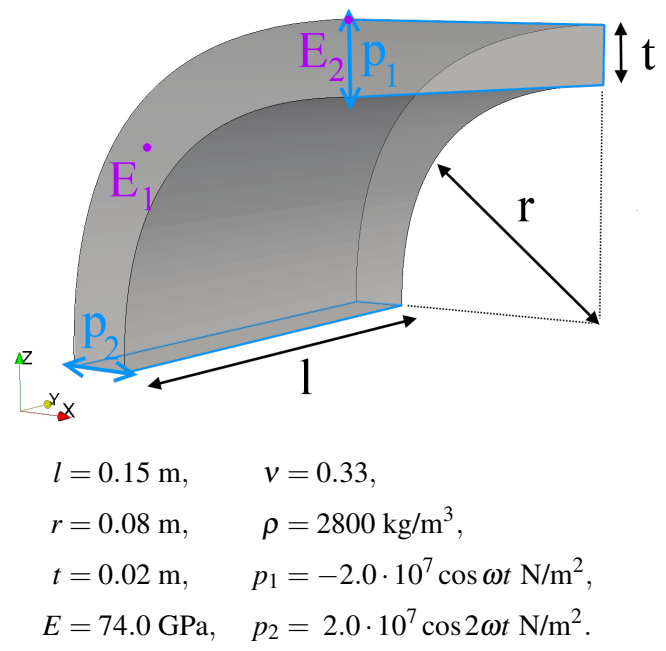

Fig. 8: Geometry, material parameters and loads of the vibrating thick cylinder

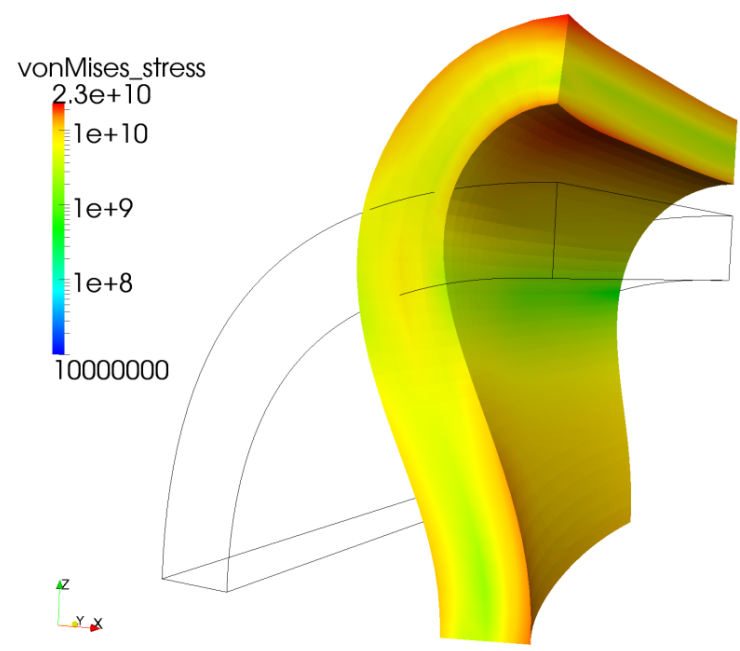

Fig. 9: Snapshot of deformation of thick cylinder at $\tau=0$ for vibration with $f / f_{1}^{h}=0.95$, colored by von Mises stress.

tions occur, the isogeometric harmonic balance still shows a good convergence behaviour, with 4-5 Newton iterations per frequency step.

In Figure 10 the $z$-amplitudes evaluated at the center point of the front surface of the cylinder $E_{1}$ are plotted. We can find a typical nonlinear resonance behaviour with two branches. For the left one we have no more convergence at $f / f_{1}^{h}=0.97$, probably due to a turning point that we can not detected with simple frequency increments. Further away from the resonance at 1.0, where $a_{1}$ from linear DFR tends to $\infty$, amplitudes of DFR and HBM correspond quite well, but then resonance behaviour becomes different and we can also detect strong contributions of other harmonics $a_{0}, a_{2}$ and $a_{3}$.

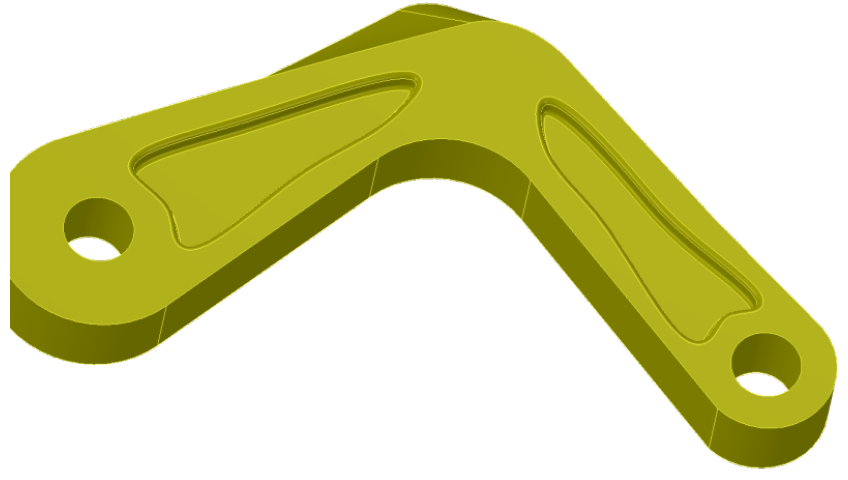

Fig. 12: Geometry of the "TERRIFIC Demonstrator" in CAD system.

For a comparison of reduction methods with the "exact" HBM solutions, we have changed the refinement of isogeometric parameterization to $p=(2,2,2), \ell=(4,4,1)$, $n=(6,6,3), N=324$ and the evaluation point to the top right corner of the front surface $E_{2}$, compare Figure 8

We compare the frequency response of the cylinder at $f / f_{1}^{h} \approx 1.0$ for full harmonic balance (HBM), modal reduction (MR) with $r=50$ and modal derivatives (MD) with $r_{d}=10(r=65)$ in Figure 11 . For modal reduction we have no meaningful reproduction of the results whatsoever, while the extended basis with modal derivatives reproduces the amplitudes of the full harmonic balance with high accuracy up to a level where strong resonance occurs.

\subsection{Large-scale application: the TERRIFIC Demonstrator}

The so-called "TERRIFIC part" is a structure which was introduced within the European project "TERRIFIC" [36] as a demonstrator for the isogeometric CAE workflow from design, over analysis to manufacturing. It was designed in a CAD system (Figure 12), an IGA-suitable NURBS volume parameterization was generated for mechanical simulation (Figure 13, and other models for dip-paint simulation and computer-aided manufacturing were derived. Here we want to use it as a realistic large-scale application for our nonlinear frequency analysis framework.

The isogeometric volume parameterization consits of 15 patches of quadratic B-Spline volumes, with a total of 6,474 control points and 19,422 DOFs. Including interface constraints on the patches, the isogeometric finite element discretization of the model has 22,914 DOFs.

The material parameters of the part, using the St. VenantKirchoff material law, are then chosen as follows:

$E=74.0 \mathrm{GPa}, \quad v=0.33, \quad \rho=2800 \mathrm{~kg} / \mathrm{m}^{3}$.

As boundary conditions we take a clamping of the right hole in Figure 13 by a zero Dirichlet condition and the periodic 

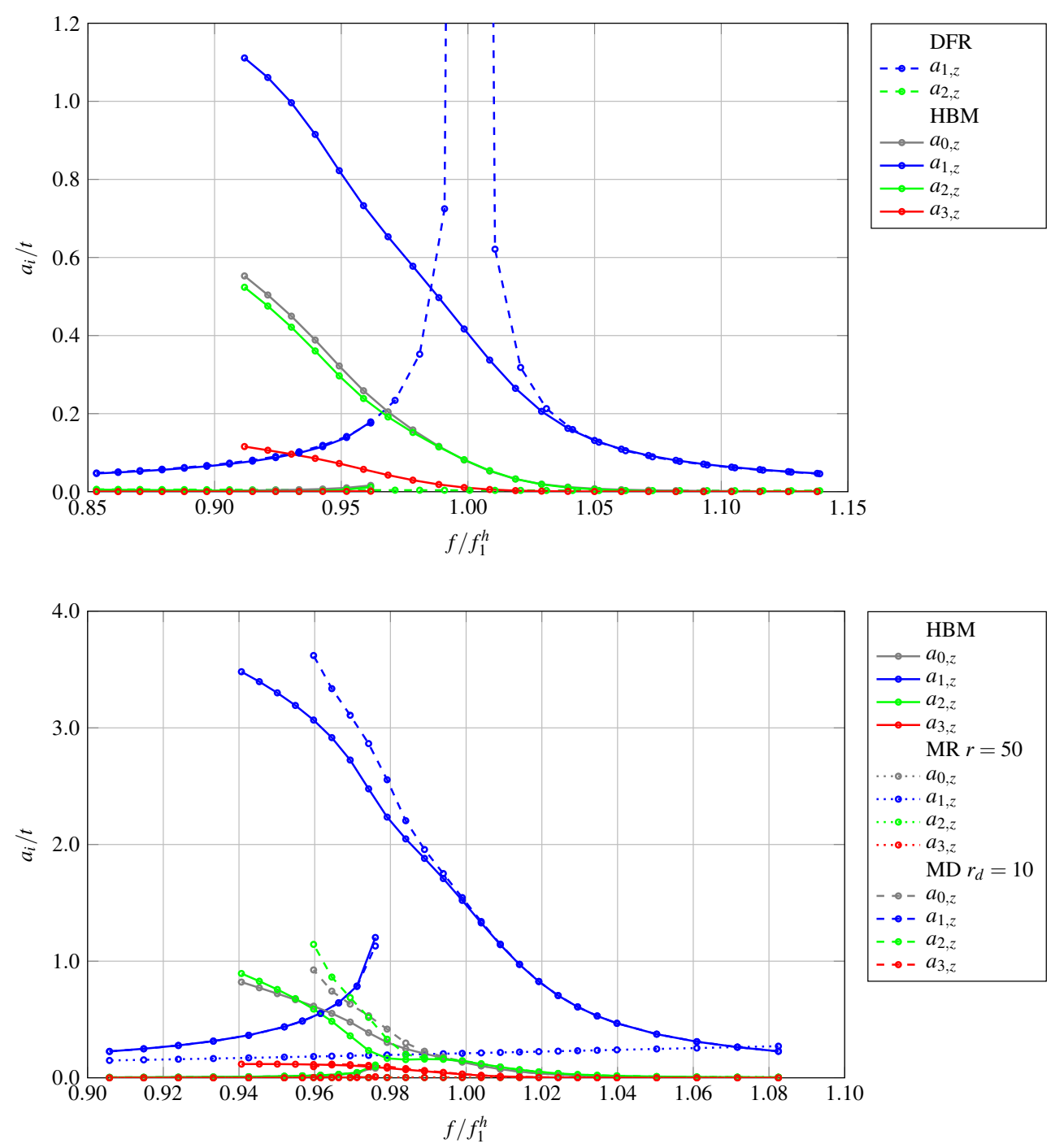

Fig. 10 Frequency response curves of $z$-amplitudes at center point of front surface $E_{1}$ for $m=3$. Comparison of DFR and HBM

Fig. 11 Frequency response curves of $z$-amplitudes of vibrating cylinder at top right point of front surface $E_{2}$ for $m=3$. Comparison of full $\mathrm{HBM}$ and HBM with MR and MD

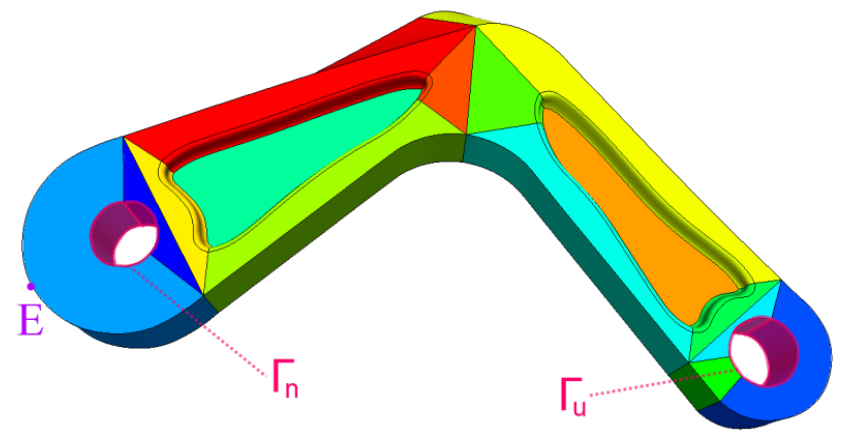

Fig. 13: IGA-suitable multi-patch volume parameterization of the "TERRIFIC Demonstrator".

excitation acts as a surface traction on the left hole (Neumann boundary condition):

$$
\mathbf{u}=\mathbf{0} \quad \text { on } \Gamma_{u},
$$

$\mathbf{t}=(60.0,-42.0,0.0)^{T} \cdot 10^{6} \cos \omega t \mathrm{~N} / \mathrm{m}^{2}$ on $\Gamma_{n}$.
With this spatial discretization, material parameters and boundary conditions we start the analysis by computing the first four eigenfrequencies $(f=2 \pi / \omega)$ of the part:

$f_{1}^{h}=223.66 \mathrm{~Hz}, \quad f_{2}^{h}=358.05 \mathrm{~Hz}$,

$f_{3}^{h}=740.78 \mathrm{~Hz}, \quad f_{4}^{h}=1233.54 \mathrm{~Hz}$.

We are interested in carrying out a frequency response analysis of the part around the first two eigenfrequencies, i.e. in the range of $150 \mathrm{~Hz}<f<450 \mathrm{~Hz}$. The surface tractions specified in 65) cause a large deformation of the part and thus we expect a significant difference between the results of a linear DFR and the nonlinear HBM.

Taking a Fourier series length of $m=3$, the problem size of harmonic balance grows in this case to a total of 160,398 DOFs and due to its low sparsity it is not solvable on a personal computer. Therefore we need to apply the reduction proposed in Section 4 to make the problem resp. the linear system solvable. 


\begin{tabular}{c|c|rr|rr} 
& full & \multicolumn{2}{|c|}{$r_{d}=5$} & \multicolumn{2}{c}{$r_{d}=10$} \\
& abs. val. & abs. val. & rel. err. & abs. val. & rel. err. \\
\hline$u_{x}$ & $1.42 \mathrm{E}-02$ & $1.33 \mathrm{E}-02$ & $5.7 \%$ & $1.41 \mathrm{E}-02$ & $0.04 \%$ \\
$u_{y}$ & $6.57 \mathrm{E}-03$ & $5.85 \mathrm{E}-03$ & $11.0 \%$ & $6.59 \mathrm{E}-03$ & $0.27 \%$ \\
$u_{z}$ & $1.05 \mathrm{E}-03$ & $2.69 \mathrm{E}-03$ & $155.8 \%$ & $1.05 \mathrm{E}-03$ & $0.48 \%$ \\
$L^{2}$ & $1.53 \mathrm{E}-04$ & $1.45 \mathrm{E}-04$ & $11.8 \%$ & $1.53 \mathrm{E}-04$ & $0.24 \%$ \\
$H^{1}$ & $1.47 \mathrm{E}-03$ & $1.40 \mathrm{E}-03$ & $8.4 \%$ & $1.47 \mathrm{E}-03$ & $0.80 \%$
\end{tabular}

Table 1: Nonlinear static analysis of "TERRIFIC Demonstrator". Comparison of full problem and reduction with modal derivatives.

As part of pre-processing we compute the nonlinear static displacement caused by a static load of the same magnitude. Then we compute the reduction basis with 5 resp. 10 linear eigenmodes and all corresponding modal derivatives, and solve the reduced versions of the nonlinear static problem. A comparison of absolute values and relative errors of displacements at an evaluation point on the very left of the structure (which we also take for plotting frequency response curves), $L^{2}$ - and $H^{1}$-norms in Table 1 reveals that $r_{d}=5$ is not sufficient to capture the nonlinear displacement behaviour, whereas $r_{d}=10$ provides a sufficient accuracy of $\left\|u_{\text {full }}-u_{r_{d}}\right\|_{L^{2}} /\left\|u_{\text {full }}\right\|_{L^{2}}<1.0 \%$.

We proceed with the harmonic balance frequency response analysis in conjunction with modal derivative reduction with $r_{d}=10$, i.e. the first 10 linear eigenmodes and the $r_{d}\left(r_{d}+1\right) / 2=55$ corresponding modal derivatives. In Figures 14, 15, 16 we have plotted the frequency response curves of $x$-, $y$ - and $z$-amplitudes evaluated at evaluation point $E$ on the left outer boundary of the "TERRIFIC part" for the frequency range $150 \mathrm{~Hz}<f<450 \mathrm{~Hz}$, togehter with corresponding amplitudes computed from linear DFR. Around $f=159.0 \mathrm{~Hz}=f_{2}^{h} / 2$ there is a remarkable sub-harmonic response in $a_{2}$, which can not be determined with linear frequency analysis. In the vicinity of the first eigenfrequency $f_{1}^{h}=223.7 \mathrm{~Hz}$ we notice that the nonlinear response behaviour in $z$-amplitudes is different from the linear one obtained from DFR. The resonance behaviour around $f_{2}^{h}=$ $358.0 \mathrm{~Hz}$ is very strong and we have convergence problems with our method for $335 \mathrm{~Hz}<f<355 \mathrm{~Hz}$. There are strong contributions from higher harmonics here, which lead to much more realistic deformations as we discuss in more detail below. Rapidly growing $z$-amplitudes at resonance indicate that there might by turning points in the frequency response curves here, which we could only follow using continuation methods.

Figure 17 shows the vibrating structure at a frequency of $f=331.3 \mathrm{~Hz}$, i.e. near the first eigenfrequency of $f_{1}^{h}=$ $358.1 \mathrm{~Hz}$, where resonance with very large deformation oc- curs. Four snapshots are taken at times $\tau=0, \pi / 2, \pi, 3 \pi / 2$, displaying the deformed structure from the HBM-MD computation colored by von Mises stress in $\mathrm{Pa}$ and as references the deformed structure from DFR linear frequency analysis and undeformed structure both in gray. It becomes obvious that the nonlinear results lead to a much better conservation of volume of the structure and thus much more realistic states of deformation. Furthermore it is interesting that the bending of the structure is stronger in the nonlinear case than in the linear case for $\tau=\pi$. This can as well be observed in Figure 18, where we have plotted the $x^{-}, y-$ and z-displacement at the evaluation point over one vibration period of $\tau \in[0,2 \pi]$ for $f=331.3 \mathrm{~Hz}$ for both HBM-MD and DFR.

Altogether, the results we present for the "TERRIFIC Demonstrator" show that a modal reduction with modal derivatives makes harmonic balance nonlinear frequency response analysis feasible even for larger applications.

\section{Summary and outlook}

The aim of this paper is to present an advanced method for nonlinear frequency response analysis of large-scale applications in solid mechanics.

We have proposed to use the harmonic balance method for nonlinear steady-state frequency response of the discretized equation of motion in the frequency domain. In conjunction with a modal projection method using eigenmodes and second order modal derivatives as reduction basis, the method can be applied even to realistic applications with large spatial discretizations. For an efficient spatial discretization of the nonlinear partial differential equations arising from 3dimensional large deformation hyperelasticity we employ the isogeometric finite element method, but our approach could be applied using any spatial discretization method. As our numerical examples show, the reduction method provides a good accuracy of frequency response amplitudes and resonance behaviour, although significantly reduces the effort for numerical solution of the harmonic balance equation system. For large-scale applications harmonic balance becomes feasible only using model order reduction.

Even though the proposed reduction method makes nonlinear frequency response analysis feasible in application to 3-dimensional structural problems, it still remains a timeconsuming task. Especially for very large applications a speedup of the sampling process is necessary, where full residual and tangent stiffness have to be assembled for every sample. A complexity reduction might there be achieved by methods such as Discrete Empirical Interpolation [42]. For further industrial problems we plan to extend the method to materials with nonlinear viscoelastic properties such as rubber and contact problems. We also aim at combining nonlinear frequency analysis with shape optimization. 

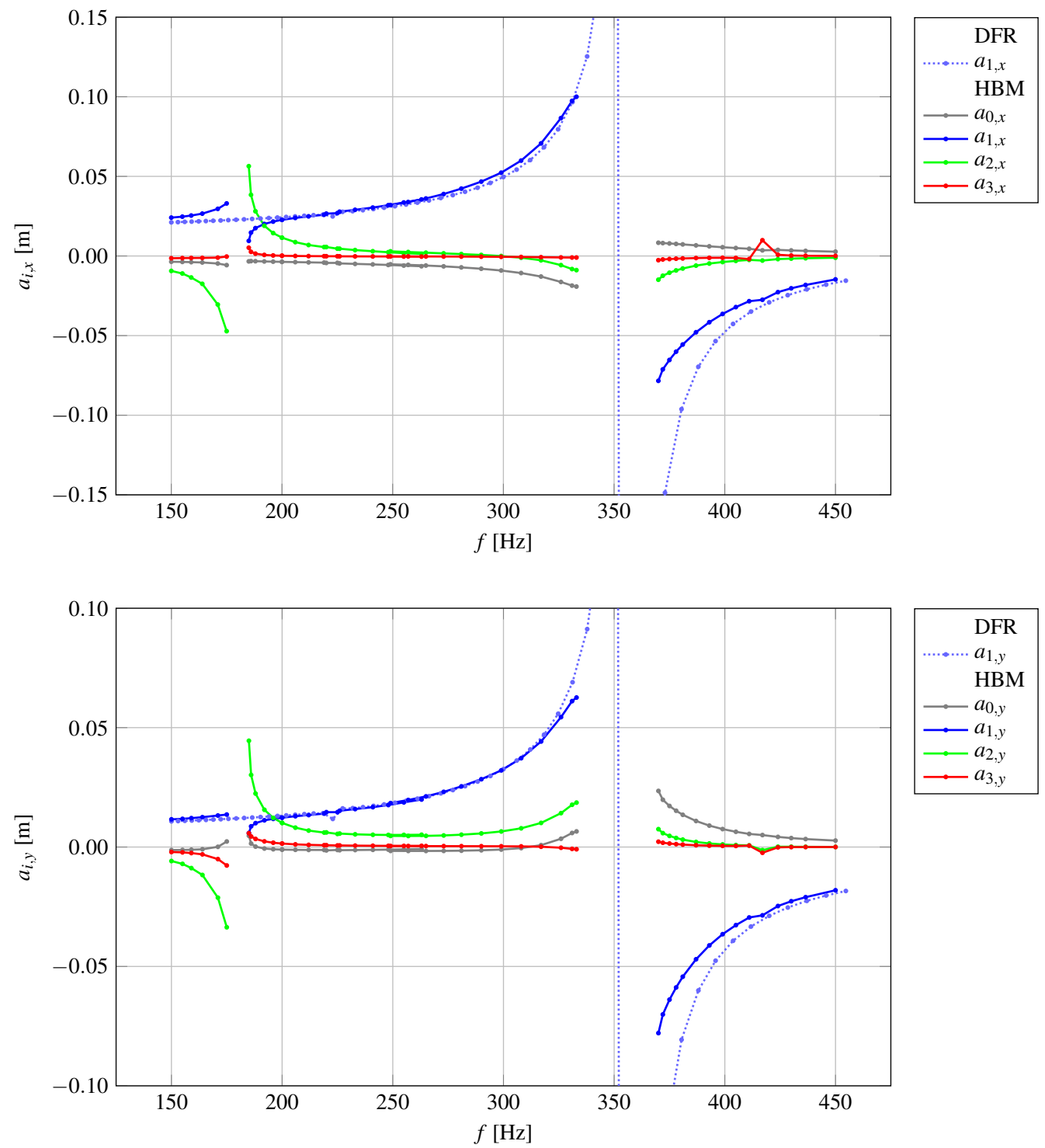

Fig. 14 Frequency response curves of $x$-amplitudes of vibrating "TERRIFIC Demonstrator" for DFR and HBM with reduction

Fig. 15 Frequency response curves of $y$-amplitudes of vibrating "TERRIFIC Demonstrator" for DFR and HBM with reduction
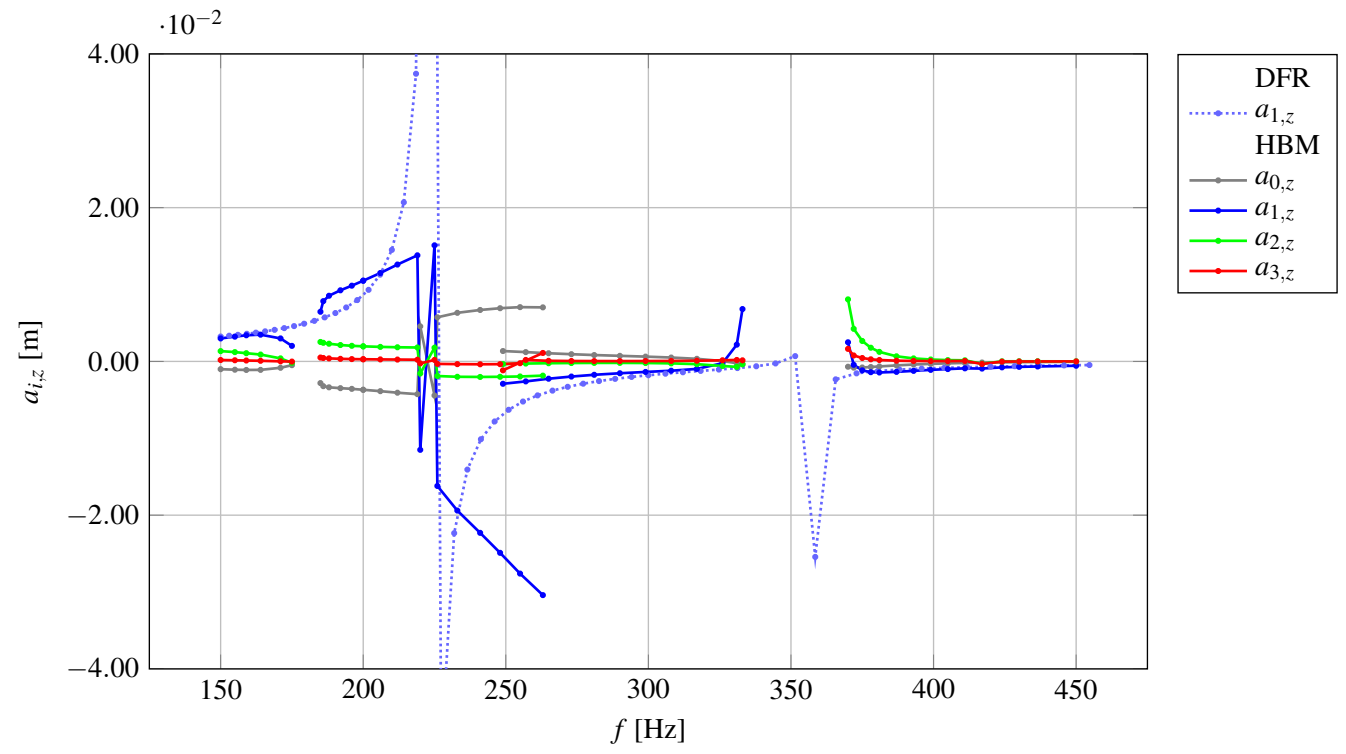

Fig. 16 Frequency response curves of $z$-amplitudes of vibrating "TERRIFIC Demonstrator" for DFR and $\mathrm{HBM}$ with reduction 


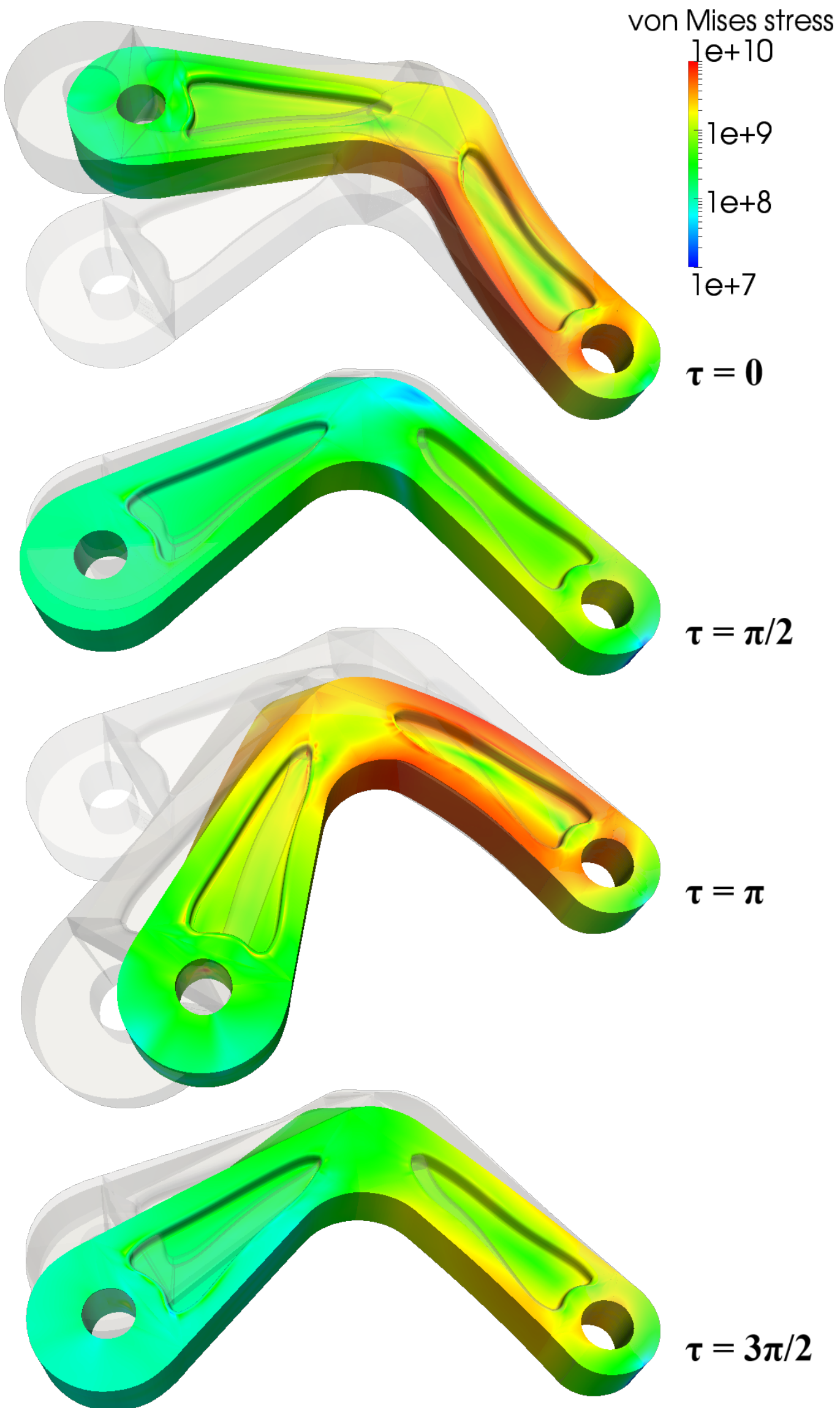

Fig. 17 Snapshots of vibrating "TERRIFIC Demonstrator" for $f=331.3 \mathrm{~Hz}$ at $\tau=0, \pi / 2, \pi, 3 \pi / 2$. Nonlinear deformation from HBM-MD is colored by von Mises stress in $\mathrm{Pa}$, linear DFR deformation and undeformed configuration are in gray 


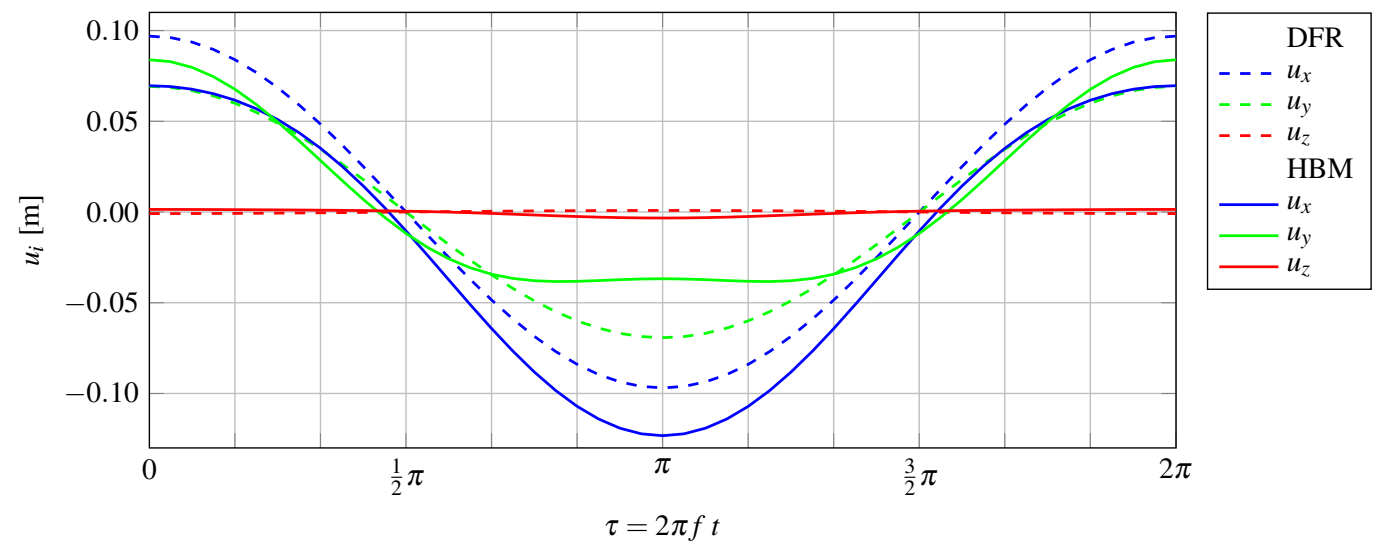

Fig. 18 Displacement of evaluation point on "TERRIFIC Demonstrator" for $f=331.3 \mathrm{~Hz}$ for HBM-MD and DFR
Acknowledgements This work is supported by the European Union within the FP7-project TERRIFIC: Towards Enhanced Integration of Design and Production in the Factory of the Future through Isogeometric Technologies [36].

The "TERRIFIC part" was designed by Stefan Boschert (Siemens AG, Germany) and the isogeometric parameterization provided by Vibeke Skytt (SINTEF, Norway).

\section{References}

1. A.H. Nayfeh and B. Balachandran. Applied Nonlinear Dynamics: Analytical Computational, and Experimental Methods. Wiley Series in Nonlinear Science. John Wiley \& Sons, 1995.

2. A.H. Nayfeh and D.T. Mook. Nonlinear Oscillations. Wiley Classics Library. John Wiley \& Sons, 1995.

3. W. Szemplinska-Stupnicka. The Behaviour of Nonlinear Vibrating Systems. Kluwer Academic Publishers, Dordrecht Boston London, 1990

4. O. Weeger, U. Wever, and B. Simeon. Isogeometric analysis of nonlinear euler-bernoulli beam vibrations. Nonlinear Dynamics, 72(4):813-835, 2013.

5. P. Wriggers. Nonlinear Finite Element Methods. Springer, 2008.

6. T. Belytschko, W. K. Liu, and B. Moran. Nonlinear Finite Elements for Continua and Structures. John Wiley \& Sons, 2000.

7. Cadence Design Systems Inc. Rf analysis in virtuoso spectre circuit simulator xl datasheet. Technical report, 2007.

8. M. Schneider, U. Wever, and Q. Zheng. Parallel harmonic balance. VLSI 93, Proceedings of the IFIP TC10/WG 10.5 International Conference on Very Large Scale Integration, Grenoble, France, 7-10 September, 1993, pages 251-260, 1993.

9. R. Lewandowski. Non-linear, steady-state vibration of structures by harmonic balance/finite element method. Computers \& Structures, 44(1-2):287-296, 1992.

10. R. Lewandowski. Computational formulation for periodic vibration of geometrically nonlinear structures, part 1: Theoretical background; part 2: Numerical strategy and examples. International Journal of Solids and Structures, 34(15):1925-1964, 1997.

11. P. Ribeiro and M. Petyt. Non-linear vibration of beams with internal resonance by the hierarchical finite element method. Journal of Sound and Vibration, 224(15):591-624, 1999.

12. P. Ribeiro. Hierarchical finite element analyses of geometrically non-linear vibration of beams and plane frames. Journal of Sound and Vibration, 246(2):225-244, 2001.

13. P. Ribeiro. Non-linear forced vibrations of thin/thick beams and plates by the finite element and shooting methods. Computers and Structures, 82(17-19):1413-1423, 2004.
14. T.J.R. Hughes. The Finite Element Method: Linear Static and Dynamic Finite Element Analysis. Dover Publications, Mineola, New York, 2000.

15. Z.-Q. Qu. Model Order Reduction Techniques with Applications in Finite Element Analysis. Springer, 2004.

16. Malte Krack, Lars Panning von Scheidt, and Jörg Wallaschek. A method for nonlinear modal analysis and synthesis: Application to harmonically forced and self-excited mechanical systems. Journal of Sound and Vibration, 332(25):6798-6814, 2013.

17. S. R. Idelsohn and A. Cardona. A reduction method for nonlinear structural dynamics analysis. Comput. Methods Appl. Mech. Engrg., 49:253-279, 1985.

18. P. M. A. Slaats, J. de Jongh, and A. A. H. J. Sauren. Model reduction tools for nonlinear structural dynamics. Computers \& Structures, 54(6):1155-1171, 1995.

19. J. Barbic. Real-time Reduced Large-Deformation Models and Distributed Contact for Computer Graphics and Haptics. $\mathrm{PhD}$ thesis, Carnegie Mellon University, 2007.

20. J. Barbic. Fem simulation of $3 \mathrm{~d}$ deformable solids: A practitioner's guide to theory, discretization and model reduction. part 2: Model reduction. In SIGGRAPH 2012 Course Notes, 2012.

21. H. Spiess. Reduction Methods in Finite Element Analysis of Nonlinear Structural Dynamics. PhD thesis, Universität Hannover, 2005.

22. J. Remke and H. Rothert. Eine modale reduktionsmethode zur geometrisch nichtlinearen statischen und dynamischen finiteelement-berechnung. Archive of Applied Mechanics, 63(2):101 $115,1993$.

23. Oleg Roderick, Mihai Anitescu, and Paul Fischer. Polynomial regression approaches using derivative information for uncertainty quantification. Nuclear Science and Engineerin, 164(2):122-139, 2010.

24. T.J.R. Hughes, J.A. Cottrell, and Y. Bazilevs. Isogeometric analysis: Cad, finite elements, nurbs, exact geometry and mesh refinement. Computer Methods in Applied Mechanics and Engineering, 194(39-41):4135-4195, 2005.

25. L.A. Piegl and W. Tiller. The Nurbs Book. Monographs in Visual Communication. Springer, 1997.

26. R.N. Simpson, S.P.A. Bordas, J. Trevelyan, and T. Rabczuk. A two-dimensional isogeometric boundary element method for elastostatic analysis. Computer Methods in Applied Mechanics and Engineering, 209-212:87-100, 2012.

27. F. Auricchio, L. Beirão da Veiga, T.J.R. Hughes, A. Reali, and G. Sangalli. Isogeometric collocation methods. Mathematical Models and Methods in Applied Sciences, 20(11):2075-2107, 2010.

28. Ch. Heinrich, B. Simeon, and S. Boschert. A finite volume method on nurbs geometries and its application in isogeometric fluid-structure interaction. Mathematics and Computers in Simulation, 82(9):1645-1666, 2012. 
29. J.A. Cottrell, T.J.R. Hughes, and Y. Bazilevs. Isogeometric Analysis: Toward Integration of CAD and FEA. John Wiley \& Sons, Ltd, 2009.

30. J.A. Cottrell, A. Reali, Y. Bazilevs, and T.J.R. Hughes. Isogeometric analysis of structural vibrations. Computer Methods in Applied Mechanics and Engineering, 195(41-43):5257-5296, 2006.

31. T.J.R. Hughes, J.A. Evans, and A. Reali. Finite element and nurbs approximations of eigenvalue, boundary-value, and initial-value problems. Computer Methods in Applied Mechanics and Engineering, 272:290-320, 2014.

32. T. Elguedj, Y. Bazilevs, V. M. Calo, and T. J. R. Hughes. B-bar and f-bar projection methods for nearly incompressible linear and non-linear elasticity and plasticity based on higher-order nurbs elements. Computer Methods in Applied Mechanics and Engineering, 197:2732-2762, 2008.

33. F. Auricchio, L. Beirão da Veiga, C. Lovadina, and A. Reali. The importance of the exact satisfaction of the incompressibility constraint in nonlinear elasticity: mixed fems versus nurbs-based approximations. Computer Methods in Applied Mechanics and Engineering, 199:314-323, 2010.

34. K. M. Mathisen, K. M. Okstad, T. Kvamsdal, and S. B. Raknes. Isogeometric analysis of finite deformation nearly incompressible solids. Rakenteiden Mekaniikka (Journal of Structural Mechanics), 44(3):260-278, 2011.

35. R. Kolman. Isogeometric free vibration of an elastic block. Engineering Mechanics, 19(4):279-291, 2012.

36. Terrific project, October 2011.

37. C. Touze and M. Amabili. Nonlinear normal modes for damped geometrically nonlinear systems: Application to reduced-order modelling of harmonically forced structures. Journal of Sound and Vibration, 298:958-981, 2006.

38. C. Touze, M. Amabili, and O. Thomas. Reduced-order models for large-amplitude vibrations of shells including in-plane inertia. Comput. Methods Appl. Mech. Engrg., 197:2030-2045, 2008.

39. Polarit Apiwattanalunggarn, Steven W. Shaw, Christophe Pierre, and Dongying Jiang. Finite-element-based nonlinear modal reduction of a rotating beam with large-amplitude motion. Journal of Vibration and Control, 9:235-263, 2003.

40. U. Becker. Efficient time integration and nonlinear model reduction for incompressible hyperelastic materials. $\mathrm{PhD}$ thesis, $\mathrm{TU}$ Kaiserslautern, 2012.

41. S. Herkt. Model Reduction of Nonlinear Problems in Structural Mechanics: Towards a Finite Element Tyre Model for Multibody Simulation. PhD thesis, TU Kaiserslautern, 2008.

42. S. Chaturantabut and D.C. Sorensen. Nonlinear model reduction via discrete empirical interpolation. SIAM J. Sci. Comput., 32(5):2737-2764, 2010 